Publ. RIMS, Kyoto Univ.

12 (1976), 391-425

\title{
Théorèmes de Comparaison en Géométrie Riemannienne
}

\author{
Par
}

\author{
A. Debiard*, B. Gaveau*, E. Mazet*
}

Ce travail, qui est la suite [4] utilise systématiquement le thèorème fondamental de comparaison entre deux équations différentielles stochastiques établi par P. Malliavin dans [13] pour obtenir les estimées asymptotiques des fonctions de Green.

Le $\S I$ donne une comparaison entre les noyaux de la chaleur et les noyaux de Green d'une variété riemannienne quelconque avec les noyaux correspondants d'une variété de comparaison choisie ici à courbure constante. On en déduit en particulier la comparaison des infimums de spectres sur les boules normales de la variété avec les infimums analogues pour les variétés à courbure constante.

$\mathrm{Au} \S \mathrm{II}$, nous donnons des démonstrations très simples de certains résultats de Yau sur les fonction harmoniques $L^{2}$, de Greene et Wu sur le fait que la seule fonctions harmonique $L^{p}(1 \leqq p<+\infty)$ d'une variété simplement connexe à courbure $\leqq 0$ est 0 ; nous démontrons que sur une variété à courbure de Ricci $\geqq 0$, il n'y a pas de fonctions harmoniques bornées non constantes.

Au §III, on étudie le lien entre la propagation de la chaleur et les géodésiques d'une variété à courbure $\leqq 0$; plus précisément on établit une propriété du type de Feynman [7] mais pour la propagation de la chaleur au lieu de la propagation de l'amplitude de probabilité: soit $\left(X_{t}^{\varepsilon}\right)_{t \in[0,1]}$ le processus conditionné pour partir de $x_{0}$ à l'instant 0 et arriver en $y_{0}$ à l'instant $\varepsilon$, reparamétré par le changement de temps $t \rightarrow \varepsilon t$. Quand $\varepsilon$ tend vers 0 , ce processus converge en loi vers le mouvement uniforme sur la géodésique minimisante joignant $x_{0}$ à $y_{0}^{\prime}$ si $y_{0}^{\prime}$ n'est pas dans le

Communiqué par K. Itô, le 12 février, 1976.

* Université Paris 6, Mathématiques, 4 place Jussieu, 75005 Paris, France. 
cut-locus de $x_{0}$, et si $y_{0}$ est dans le cut-locus de $x_{0}$ vers l'une des géodésiques minimisantes (qui sont en nombre fini) avec une probabilité que, sous certaines conditions techniques, on peut calculer. La différence essentielle avec la théorie de Feynman est que la propagation de la chaleur ne se concentre que sur les géodésiques minimisantes, alors que dans la propagation de l'amplitude de probabilité les choses se passent plutôt comme si l'on avait un processus qui se concentre sur toutes les géodésiques: en particulier si $y_{0}=x_{0}$, le processus de la chaleur limite reste constamment en $x_{0}$, alors que la limite classique de l'équation de Schrödinger fait apparaitre toutes les géodésiques périodiques (la phase de chaque géodésique étant son indice de Morse).

"Une étude analogue a été effectuée dans [14] par A. S. Molchanov; Molchanov étudie la convergence en loi du processus accéléré vers la ou les géodésiques minimisantes ainsi que la fluctuation. Le travail de Molchanov concerne l'asymptotique du noyau de la chaleur pour $t \rightarrow 0^{+}$ et il obtient l'équivalent exact de $p_{t}(x, y) \quad\left(t \rightarrow 0^{+}\right)$selon la topologie de l'espace des géodésiques minimisantes joignant $x$ à $y$."

Remarque. Une petite différence entre ce travail et [4] est que nous avons considéré ici l'équation de la chaleur $\frac{\partial}{\partial t}=\frac{1}{2} \Delta$ plutôt que $\frac{\partial}{\partial t}$ $=\Delta$. Il en résulte que cetaines formules diffèrent par des facteurs puissances de $\frac{1}{2}$.

\section{Comparaison des Noyaux Fondamentaux}

1. Notations.

a) Soit $V$ une variété riemannienne complète, $K$ sa courbure sectionnelle, $\Delta$ le laplacien des fonctions sur $V$. Pour $x_{0} \in V$ et $\rho_{0}$ nombre réel strictement compris entre 0 et le rayon d'injectivité en $x_{0}$, on désigne par $B\left(x_{0}, \rho_{0}\right)$ la boule géodésique normale de centre $x_{0}$ et de rayon $\rho_{0}$. On pose pour $x \in B\left(x_{0}, \rho_{0}\right), r(x)=d\left(x_{0}, x\right)$, de sorte que $r$ est une fonction $C^{\infty} \operatorname{sur} B\left(x_{0}, \rho_{0}\right)-\left\{x_{0}\right\}$.

b) On notera $V^{a}, K^{a}, \Delta^{a}, B^{a}\left(x_{0}, \rho_{0}\right), r^{a}$ les mêmes objets mais relatifs à la variété standard de courbure constante a (c'est-à-dire $K^{a} \equiv a$ ). On observe que si $a \leqq 0$ il n'y a pas de restriction a priori sur $\rho_{0}$, mais 
si $a>0$ alors on doit avoir $\rho_{0}<\frac{\pi}{\sqrt{a}}$.

c) Pour tout choix des centres $x_{0}$ dans $V$ et $V^{a}$ et tout $\rho_{0}$ admissible on peut identifier $B\left(x_{0}, \rho_{0}\right)$ et $B^{a}\left(x_{0}, \rho_{0}\right)$ à l'aide des cartes exponentielles, identification qui est unique modulo des isométries dans les espaces tangents $T_{x_{0}} V$ et $T_{x_{0}} V^{a}$. Nous supposerons une telle identification choisie une fois pour toutes. On a alors $r^{a}(x)=r(x)$ pour tout point $x$ des boules ainsi identifiées.

Enfin, si l'on veut comparer deux variétés simplement connexes à courbure $\leqq 0$, on pourra prendre $\rho_{0}=+\infty$, et identifier globalement les deux variétés par le procédé précédent.

\section{Comparaison des noyaux de la chaleur.}

Dans la première partie du théorème qui suit $p_{t}\left(x_{0}, x\right), x \in B\left(x_{0}, \rho_{0}\right)$, désigne le noyau de la chaleur sur $B\left(x_{0}, \rho_{0}\right)$ c'est-à-dire la densité de probabilité de transition du brownien arrêté à la sortie. Dans la deuxième partie $p_{t}\left(x_{0}, x\right), x \in V$, désigne le noyau global de la chaleur sur $V$ c'està-dire la densité de probabilité de transition du brownien sur $V$.

\section{Théorème $\mathbb{1}$.}

1) Si $K \leqq a$ (resp. $b \leqq K)$ sur $B\left(x_{0}, \rho_{0}\right)$, alors pour tout $x \in B\left(x_{0}, \rho_{0}\right)$ et tout $t>0$, on $a$ :

$$
p_{t}\left(x_{0}, x\right) \leqq p_{t}{ }^{a}\left(x_{0}, x\right) \quad\left(\operatorname{resp} . p_{t}{ }^{b}\left(x_{0}, x\right) \leqq p_{t}\left(x_{0}, x\right)\right) .
$$

2) Si $V$ est simplement connexe et si $K \leqq-a^{2} \leqq 0$ (resp. $\left.-b^{2} \leqq K \leqq 0\right)$ sur $V$ alors, pour tout $x \in V$ et tout $t>0$, on $a$ :

$$
p_{t}\left(x_{0}, x\right) \leqq p_{t}^{\left(-a^{2}\right)}\left(x_{0}, x\right) \quad\left(\text { resp. } p_{t}^{\left(-b^{2}\right)}\left(x_{0}, x\right) \leqq p_{t}\left(x_{0}, x\right)\right) .
$$

\section{Démonstration.}

1. Nous supposerons $x \neq x_{0}$, le cas $x=x_{0}$ en résultant par continuité des $p_{t}$.

Soit $\varepsilon$ tel que $0<\varepsilon<\rho_{0}, x \notin B\left(x_{0}, \varepsilon\right)$. Nous noterons $\left(X_{t}\right)_{t \in R_{+}}$le mouvement brownien issu de $x$ sur $V$, par $\left(X_{t}^{a}\right)_{t \in R}$ le mouvement brownien issu de $x$ sur $V^{a}$, par $(\Omega, P)$ et $\left(\Omega^{a}, P^{a}\right)$ les espaces de probabilité respectifs de ces processus, par $T_{\rho_{0}}$ et $T_{\rho_{0}}^{a}$ leurs temps respectifs de sortie 
de la boule $B\left(x_{0}, \rho_{0}\right)$. Nous établirons d'abord le lemme suivant.

Lemme 1. $P\left(t<T_{\rho_{0}}, X_{t} \in B\left(x_{0}, \varepsilon\right)\right) \leqq P^{a}\left(t<T_{\rho_{0}}^{a}, X_{t}^{a} \in B\left(x_{0}, \varepsilon\right)\right)$.

Démonstration du lemme. Elle est semblable à celle du théorème 1 de [4], à ceci près qu'il faut étudier, en plus des temps de sortie de la grande boule $B\left(x_{0}, \rho_{0}\right)$, la position au temps $t$ par rapport à la petite boule $B\left(x_{0}, \varepsilon\right)$ :

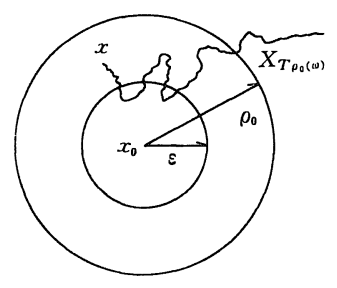

Désignons par $\theta, \theta^{a}$ les densités de volume en coordonnées polaires géodésiques de centre $x_{0}$, $\theta^{\prime}$ et $\left(\theta^{a}\right)^{\prime}$ leurs dérivées par rapport à $r$. Les fonctions $\frac{\theta^{\prime}}{\theta}$ et $\frac{\left(\theta^{a}\right)^{\prime}}{\theta^{a}}$ n'ont de singularité qu'en $x_{0}$ où presque sûrement $X_{t}$ et $X_{t}^{a}$ ne passent pas (nous écartons le cas de la dimension 1 qui ne nous intéresse pas ici). On a alors presque sûrement (formule de Itô):

$$
\begin{aligned}
& \left(\operatorname{ro} X_{t}^{a}\right)(\omega)=r(x)+b_{t}\left(\omega^{\prime}\right)+\frac{1}{2} \int_{0}^{t} \frac{\left(\theta^{a}\right)^{\prime}}{\theta}\left(X_{s}^{a}(\omega)\right) d s \\
& \left(\operatorname{ro} X_{t}\right)(\omega)=r(x)+b_{t}\left(\omega^{\prime \prime}\right)+\frac{1}{2} \int_{0}^{t} \frac{\theta^{\prime}}{\theta}\left(Y_{s}(\omega)\right) d s
\end{aligned}
$$

où $b_{t}$ est un brownien standard sur un certain espace de probabilité $\left(\Omega_{1}\right.$, $\left.P_{1}\right)$ et $\omega \rightarrow \omega^{\prime}, \omega \rightarrow \omega^{\prime \prime}$ des applications de $(\Omega, P)$ et $\left(\Omega^{a}, P^{a}\right)$ respectivement dans $\left(\Omega^{1}, P^{1}\right)$, respectant les mesures.

Soit $z_{t}^{a}\left(\omega_{1}\right)$ le processus défini sur $\left(\Omega^{1}, P^{1}\right)$ par la résolution du problème de Cauchy suivant:

$$
\frac{d z_{t}^{a}\left(\omega_{1}\right)}{d t}=\frac{1}{2} \frac{\left(\theta^{a}\right)^{\prime}}{\theta}\left(r(x)+b_{t}\left(\omega_{1}\right)+z_{t}^{a}\left(\omega_{1}\right)\right), z_{0}^{a}\left(\omega_{1}\right)=0 .
$$

Par (1) on a presque sûrement: 


$$
\left(\operatorname{ro} X_{t}^{a}\right)(\omega)=r(x)+b_{t}\left(\omega^{\prime}\right)+z_{t}^{a}\left(\omega^{\prime}\right) .
$$

D'autre part la condition de courbure $K \leqq a$ entraîne $\frac{\theta^{\prime}}{\theta} \geqq \frac{\left(\theta^{a}\right)^{\prime}}{\theta^{a}}$ sur $B\left(x_{0}, \rho_{0}\right)$. Alors, d'après (2) utilisant comme en [4] le lemme de comparaison de P. Malliavin [13], on voit que presque sûrement on a

$$
\left(\text { ro } X_{t}\right)(\omega) \geqq r(x)+b_{t}\left(\omega^{\prime \prime}\right)+z_{t}^{a}\left(\omega^{\prime \prime}\right) .
$$

On déduit de là que:

$$
\begin{aligned}
& P(t<\left.T_{\rho_{0}}(\omega), X_{t}(\omega) \in B\left(x_{0}, \varepsilon\right)\right)=P\left(\left(\operatorname{ro} X_{s}\right) \quad(\omega)<\rho_{0} \forall s \in[0, t],\right. \\
&\left.\quad\left(\operatorname{ro} X_{t}\right)(\omega)<\varepsilon\right) \\
& \leqq P\left(r(x)+b_{s}\left(\omega^{\prime \prime}\right)+z_{s}{ }^{a}\left(\omega^{\prime \prime}\right)<\rho_{0} \quad \forall s \in[0, t], r(x)+b_{t}\left(\omega^{\prime \prime}\right)\right. \\
&\left.+z_{t}^{a}\left(\omega^{\prime \prime}\right)<\varepsilon\right) \\
&= P^{1}\left(r(x)+b_{s}\left(\omega_{1}\right)+z_{s}{ }^{a}\left(\omega_{1}\right)<\rho_{0} \forall s \in[0, t], r(x)+b_{t}\left(\omega_{1}\right)\right. \\
&\left.+z_{t}^{a}\left(\omega_{1}\right)<\varepsilon\right) \\
&= P^{a}\left(r(x)+b_{s}\left(\omega^{\prime}\right)+z_{s}^{a}\left(\omega^{\prime}\right)<\rho_{0} \forall s \in[0, t], r(x)+b_{t}\left(\omega^{\prime}\right)\right. \\
&\left.+z_{t}^{a}\left(\omega^{\prime}\right)<\varepsilon\right) \\
&= P^{a}\left(\left(\operatorname{ro} X_{s}^{a}\right)(\omega)<\rho_{0} \forall s \in[0, t], \quad\left(\operatorname{ro~} X_{t}^{a}\right)(\omega)<\varepsilon\right) \\
&= P^{a}\left(t<T_{\rho_{0}}^{a}(\omega), X_{t}^{a}(\omega) \in B(x, \varepsilon)\right)
\end{aligned}
$$

ce qui établit le lemme 1.

Fin de la démonstration du théorème 1.

On a par définition

$$
\begin{aligned}
& \int_{B\left(x_{0}, \varepsilon\right)} P_{t}(y, x) d y=P\left(t<T_{\rho_{0}}(\omega), \quad X_{t}(\omega) \in B\left(x_{0}, \varepsilon\right)\right) \\
& \int_{B\left(x_{0}, \varepsilon\right)} P_{t}^{a}(y, x) d^{a} y=P\left(t<T_{\rho_{0}}^{a}(\omega), \quad X_{t}^{a}(\omega) \in B\left(x_{0}, \varepsilon\right)\right) .
\end{aligned}
$$

Le lemme 1 dit donc que $\int_{B\left(x_{0}, \varepsilon\right)} P_{t}(y, x) d y \leqq \int_{B\left(x_{0}, \varepsilon\right)} P_{t}^{a}(y, x) d^{a} y$.

D'autre part la condition de courbure $K \leqq a$ entraine que $\operatorname{Vol} B\left(x_{0}, \varepsilon\right)$ $\geqq \operatorname{Vol}^{a} B^{a}\left(x_{0}, \varepsilon\right)$ d'où

$$
\frac{1}{\operatorname{Vol} B\left(x_{0}, \varepsilon\right)} \int_{B\left(x_{0}, \varepsilon\right)} P_{t}(y, x) d x \leqq \frac{1}{\operatorname{Vol}^{a} B\left(x_{0}, \varepsilon\right)} \int_{B\left(x_{0}, \varepsilon\right)} P_{t}^{a}(y, x) d^{a} y .
$$

En faisant tendre $\varepsilon$ vers 0 on a à la limite, par continuité des $P_{t}$ : 


$$
P_{t}\left(x_{0}, x\right) \leqq P_{t}^{a}\left(x_{0}, x\right) \text {. }
$$

L'inégalité correspondant au cas $b<K$ se démontre de même en untilisant les inégalités $\frac{\left(\theta^{b}\right)^{\prime}}{\theta^{b}} \geqq \frac{\theta^{\prime}}{\theta}, \operatorname{Vol}^{b} B\left(x_{0}, \varepsilon\right) \geqq \operatorname{Vol} B\left(x_{0}, \varepsilon\right)$.

2. La seconde partie se démontre de la même manière, avec seulement une simplification, du fait qu'il n'y a pas à considérer de temps de sortie $\mathrm{T}_{\rho_{0}}\left(\rho_{0}=+\infty\right)$. Il faudra toutefois, pour que ce que l'on écrit ait un sens, qu'il n'y ait pas explosion du brownien. Cette condition sera remplie par exemple si la courbure est uniformément minorée (cf. théorème 2 de [4]).

Exemple 1. Pour une variété de dimension $n$, simplement connexe, à courbure $\leqq 0$, sans explosion, on a

$$
P_{t}\left(x_{0}, x\right) \leqq(2 \pi t)^{-n / 2} e^{-r^{2 / 2 t}} \quad r=d\left(x_{0}, x\right) .
$$

Exemple 2. Pour une variété de dimension 3, simplement connexe, à courbure $K \leqq-a^{2}$, sans explosion (resp. à courbure $K$ satisfaisant $-b^{2}$ $\leqq K \leqq 0$ ), on a

$$
P_{t}\left(x_{0}, x\right) \leqq(2 \pi t)^{-3 / 2} e^{-a^{2} t / 2} e^{-r^{2} / 2 t} \frac{a r}{\text { Sh } a r}
$$

(resp. $\left.\quad P_{t}\left(x_{0}, x\right) \geqq(2 \pi t)^{-3 / 2} e^{-b 2 t / 2} e^{-r^{2} / 2 t} \frac{b r}{\operatorname{Sh} b r}\right)$.

Démonstration. Il s'agit simplement de calculer le noyau de la chaleur en dimension 3 pour l'espace à courbure constante $-a^{2}$; pour cela on procède par approximations successives en utilisant la technique de [2], [3]: on cherche la solution sous la forme

$$
(2 \pi t)^{-3 / 2} e^{-r^{2 / 2 t}}\left(u_{0}(x, y)+t u_{1}(x, y)+\cdots+t^{k} u_{k}(x, y)+\cdots\right) .
$$

Comme il n'y a pas d'ennuis topologiques et qu'on peut obtenir des expressions explicites des $u_{k}$, il n'est pas besoin d'utiliser les fonctions plateaux et les convolutions sur l'espace temps: on cherche directement une série qui soit localement uniformément convergente avec ses dérivées jusqu'à l'ordre 2, et qui satisfasse formellement l'équation de la chaleur. Les $\imath \iota_{k}$ fonctions de $r$ seul, sont donnés par la même loi récursive que 
dans le cas compact:

$$
\begin{gathered}
u_{0}=\theta^{-1 / 2} \\
u_{k+1}(r)=\frac{1}{2} \frac{(\theta(r))^{-1 / 2}}{r^{k+1}} \int_{0}^{r}(\theta(s))^{1 / 2} \Delta u_{k}(s) s^{k} d s .
\end{gathered}
$$

Ici $u_{0}(r)=\frac{a r}{\text { Sh } a r}$, d'où l'on calcule de proche en proche

$$
u_{k}(r)=\frac{(-1)^{k}}{k !} \frac{a^{2 k}}{2^{k}} u_{0}(r), \quad \Delta u_{k}(r)=\frac{(-1)^{k+1}}{(k+1) !} \frac{a^{2 k+2}}{2^{k}} u_{0}(r)
$$

ce qui fournit le résultat.

\section{Remarque.}

1. J. Vauthier démontre des théorèmes analogues concernant le propagateur de la chaleur pour les formes différentielles [14].

2. Dans les applications du théorème 1 il peut être intéressant d'aroir autant que possible une expression explicite du propagateur de la chaleur en courbure constante. Dans le cas simplement connexe à courbure constante $-a^{2} \leqq 0$, soit $P_{t}^{(n)}(r)$ ce propagateur (qui ne dépend que du temps et de la distance $r$ ). Millson a établi la relation de récurrence suivante (non publié).

$$
P_{i}^{(n+2)}(r)=-\frac{1}{2 \pi} e^{-n a 2 \frac{t}{2}} \frac{1}{a \operatorname{Sh} a r} \frac{d}{d r} P_{t}^{(n)}(r),
$$

ce qui ramène à calculer $P_{t}^{(n)}$ pour $n=2,3$. Pour $n=3$, ce calcul a été fait à l'exemple 2 ci-dessus. Pour $n=2$, il a été donné par MacKean $[12]$ :

$$
P_{t}^{(2)}(r)=a \frac{c^{-a^{2} t / 8}}{(2 \pi t)^{3 / 2}} \int_{r}^{\omega} \frac{e^{-\rho^{2} / 2 t} \rho d \rho}{\sqrt{\mathrm{Ch} a \rho-\mathrm{Ch} a r}}
$$

3. Applications: comparaison des plus petites valeurs propres; comparaison des fonctions de Green.

\section{Théorème 2.}

1. Soit $\lambda_{1}$ la premiére valeur propre du laplacien - d sur les fonctions $L^{2}\left(B\left(x_{0}, \rho_{0}\right)\right)$ nulles au bord de la boule $\left(\rho_{0}<+\infty\right)$, et $\lambda_{1}{ }^{a}$ la 
même quantité relative à la courbure constante a. Alors si $K \leqq a$ sur $B\left(x_{0}, \rho_{0}\right)$ (resp. si $K \geqq b$ sur $\left(B\left(x_{0}, \rho_{0}\right)\right)$ on a $\lambda_{1}^{a} \leqq \lambda_{1} \quad$ (resp. $\left.\lambda_{1}^{b} \geqq \lambda_{1}\right)$.

2. Si $V$ est simplement connexe à courbure $K \leqq-a^{2}$, la première valeur propre sur les fonction $L^{2}(V)$ est au moins égale à $\frac{(n-1)^{2} a^{2}}{4}$.

Démonstration. Nous nous appuierons sur le lemme suivant (qui fait partie du folklore concernant les fonctions propres du laplacien, mais dont nous n'avons pas vu de preuve dans la littérature):

Lemme 2. Soit $\Omega$ un domaine borné d'une varieté riemannienne, dont le bord $\partial \Omega$ soit une hypersurface de classe $C^{1}$. Soit $\lambda_{1}$ la première valeur propre de $-\Delta$ sur $\Omega$ au sens du théorème 1 . Alors $\lambda_{1}$ est une valeur propre simple, et il lui correspond une fonction propre qui est strictement positive sur $\Omega$.

Démonstration du lemme 2. Nous désignerons par $C$ l'espace de Banach des fonctions de classe $C^{1}$ sur l'adhérence $\bar{\Omega}$ de $\Omega$, nulles au bord. Dans $C$ nous considérerons le cône fermé $K$ des fonctions qui sont $\geqq 0$ sur $\Omega$ (d'où il résulte que leur dérivée normale intérieure est $\geqq 0$ sur $\partial \Omega)$. L'intérieur $\stackrel{\circ}{K}$ de $K$ est l'ensemble des fonctions de $C$ qui sont strictement positives sur $\Omega$ et dont la dérivée normale intérieure est strictement positive sur $\partial \Omega$.

Soit d'autre part $g$ la fonction de Green de $\Omega$, et $G$ l'opérateur inverse du laplacien:

$$
(G f)(x)=\int_{\Omega} g(x, y) f(y) d y
$$

qui est un opérateur compact de $C$ dans $C$.

Pour établir le lemme 2, il suffit d'après un théorème de KreinRutman ([10], théorème 6.3 p. 267) de montrer que pour toute fonction $f \in K$ (autre que la fonction identiquement nulle), $G f$ est dans $\stackrel{\circ}{K}$.

Que $G f$ soit strictement positive sur $\Omega$ est clair, du fait que $g$ est strictement positive sur $\Omega \times \Omega$

Pour la dérivée normale on a

$$
\frac{\partial G f}{\partial \vec{n}}(x)=\int_{\Omega} \frac{\partial g}{\partial \vec{n}}(x, y) f(y) d y=\int_{\Omega} \Pi(x, y) f(y) d y
$$


où $\Pi(x, y)$ est le noyau de Poisson de $\Omega$, lequel est strictement positif sur $\partial \Omega \times \Omega$, de sorte que $\frac{\partial G f}{\partial \vec{n}}$ est strictement positif sur $\partial \Omega$. Cela établit le lemme 2.

\section{Démonstration du théorème 2.}

1. Regardons par exemple le cas $K \leqq a$. On a

$$
P_{t}\left(x_{0}, x_{0}\right)=\sum_{i=1}^{\infty} e^{-\lambda_{i} t}\left|\Psi_{i}\left(x_{0}\right)\right|^{2}
$$

où $\left\{\Psi_{i}\right\}$ est une suite de fonctions propres normalisées orthogonales. Comme $P_{t}{ }^{a}\left(x_{0}, x_{0}\right) \geqq P_{t}\left(x_{0}, x_{0}\right)$, on a

$$
\sum_{i=1}^{\infty} e^{-\lambda_{i} t}\left|\Psi_{i}\left(x_{0}\right)\right|^{2} \leqq \sum_{i=1}^{\infty} e^{-\lambda_{i}^{a} t}\left|\Psi_{i}{ }^{a}\left(x_{0}\right)\right|^{2} .
$$

D'après le lemme $2,\left|\Psi_{1}\left(x_{0}\right)\right|^{2}$ et $\left|\Psi_{1}{ }^{a}\left(x_{0}\right)\right|^{2}$ sont des nombres strictement positifs.

Pour tout $\mu>\lambda_{1}$, multiplions les deux membres par $e^{n t}$. Alors le premier tend vers $+\infty$ quant $t$ tend vers $+\infty$, donc le second fait de même. On en conclut que $\lambda_{1}{ }^{a} \leqq \mu$ pour tout $\mu>\lambda_{1}$, d'où $\lambda_{1}{ }^{a} \leqq \lambda_{1}$.

2. est démontré dans [12]; l'intégration par parties faite dans [12] montre que $\lambda_{1}{ }^{\left(-a^{2}\right)}=\left(n-\frac{1)^{2} a^{2}}{4}\right.$. Notre estimation résulte alors du théorème 1 et de l'écriture explicite de la résolution spectrale de l'opérateur $P_{t}$ (semi-groupe de la chaleur). Il suffit d'écrire

$$
\left(P_{t} f \mid h\right)=\int_{\lambda}^{\infty} e^{-t} d\left(E_{\lambda} f \mid h\right) \quad f, h \in L^{2}(V)
$$

et de procéder comme en 1.

Remarque. 1 a été conjecturé dans [4]. 'T. Aubin [1] en a donné une démonstration indépendante qui utilise une méthode de symétrisation du type de Steiner.

Autre estimation de $\lambda_{1}$ : L'estimation donnée au théorème 2-1 est bien entendu la meilleure possible, mais elle est peu explicite. C'est pourquoi nous rappelons ici, pour le cas de la courbure majorée, une estimation plus faible, mais plus explicite, que nous avons établie dans [4] (théorème 3). 
Théorème 2 bìs. Les notations étant celles du théorème 2-1, on $a$ si $K \leqq a$ sur $B\left(x_{0}, \rho_{0}\right)$ :

$$
\lambda_{1} \geqq\left(\int_{0}^{\rho_{0}} \frac{d s}{\theta^{a}(s)} \int_{0}^{s} \theta^{a}(t) d t\right)^{-1} .
$$

Rappelons l'expression classique de $\theta^{a}$ :

$$
\theta^{a}(r)=\left\{\begin{array}{lll}
\left(\frac{1}{\sqrt{a}} \sin \sqrt{a} r\right)^{n-1} & \text { si } & a>0 \\
r^{n-1} & \text { si } & a=0 \\
\left(\frac{1}{\sqrt{-a}} \operatorname{Sh} \sqrt{-a} r\right)^{n-1} & \text { si } & a<0
\end{array}\right.
$$

Théorème 3. Soit $x_{0} \in V, g_{B\left(x_{0}, \rho_{0}\right)}\left(x_{0}, y\right)$ la fonction de Green de $B\left(x_{0}, \rho_{0}\right)$ de pôle $x_{0}$, et $g_{V}(x, y)$ la fonction de Green globale de $V$ si elle existe.

1.) Si $K \leqq a($ resp. $K \geqq b)$ on a pour tout $y \in B\left(x_{0}, \rho_{0}\right)$

$$
g_{B\left(x_{0}, \rho_{0}\right)}\left(x_{0}, y\right) \leqq g_{B^{a}\left(x_{0}, \rho_{0}\right)}^{a}\left(x_{0}, y\right)
$$

(resp. $\left.\quad g_{B\left(x_{0}, \rho_{0}\right)}\left(x_{0}, y\right) \geqq g_{B^{b}\left(x_{0}, \rho_{0}\right)}^{b}\left(x_{0}, y\right)\right)$.

2.) Supposons $V$ simplement connexe à courbure $\leqq 0$ et $K \leqq-a^{2}$ $<0 \quad$ (resp. $-b^{2} \leqq K \leqq 0$ et $n \geqq 3$ ). Alors $g_{V}(x, y) \leqq g^{-a^{2}}(x, y) \quad$ (resp. $\left.g_{V}(x, y) \geqq g^{-b 2}(x, y)\right)$.

\section{Démonstration.}

1. Dans le cas de la boule, la fonction de Green existe toujours et vaut

$$
g_{B\left(x_{0}, \rho_{0}\right)}(x, y)=\int_{0}^{\infty} P_{t}\left(x_{0}, y\right) d t
$$

où le $P_{t}$ est celui de la boule. Le résultat en découle immédiatement par le théorème 1 .

2. Pour $V$ simplement connexe à courbure $\leqq 0$ et $K \leqq-a^{2}<0$, on a

$$
g_{V}(x, y)=\int_{0}^{\infty} P_{t}(x, y) d t \leqq \int_{0}^{\infty} P_{t}^{-a^{2}}(x, y) d t
$$

la dernière intégrale convergeant absolument car $V^{-a^{2}}$ est toujours à brownien transient, et ayant pour valeur $g^{-a 2}(x, y)$. 
Lorsque $-b^{2} \leqq K \leqq 0$ et $n \geqq 3, V$ est à brownien transient et l'on peut écrire $g_{V}(x, y)=\int_{0}^{\infty} P_{t}(x, y) d t<+\infty$, d'où

$$
g_{V}(x, y) \geqq \int_{0}^{\infty} P_{t}^{-b^{2}}(x, y) d t=g^{-b^{2}}(x, y) .
$$

Remarque. Dans le cas de la boule la fonction de Green de comparaison s'écrit ( $\rho_{0}$ étant toujours soumis aux éventuelles restrictions de I.1):

$$
g_{B^{a}\left(x_{0}, \rho_{0}\right)}^{a}\left(x_{0}, y\right)=\frac{1}{\omega_{n-1}} \int_{d\left(x_{0}, y\right)}^{\rho_{0}} \frac{d r}{\theta^{a}(r)},
$$

tandis que pour $a<0$ la fonction de Green globale de $V^{a}$ est donné par:

$$
g^{a}(x, y)=\frac{1}{\omega_{n-1}} \int_{d(x, y)}^{\infty} \frac{d r}{\theta^{a}(r)},
$$

où $\omega_{n-1}$ est l'aire $(n-1)$-dimensionnelle de la sphère unité de $R^{n}$, et $\theta^{a}$ a l'expression donnée après le théorème 2 bis ci-dessus.

En effet, $g_{B^{a}\left(x_{0}, \rho_{0}\right)}^{a}$ (resp. $\left.g^{a}\right)$ ainsi définie satisfait les propriétés caractéristiques de la fonction de Green: nullité au bord (resp. à l'infini) harmonicité hors de $x_{0}$, singularité requise en $x_{0}$.

\section{Comparaison des temps moyens de sortie.}

Nous rappelons ici pour être complets des résultats déjà établis en [4] (th. 1). En appliquant le lemme de Malliavin comme dans le lemme 1 ci-dessus on voit que si $K \leqq a$ :

$$
P_{x}\left(T_{\rho_{0}}>t\right) \leqq P_{x}^{a}\left(T_{\rho_{0}}^{a}>t\right)
$$

pour tout $t>0$. L'indice inférieur $x$ attaché au symbole $P$ signifie que l'on raisonne sur le processus partout du point $x \in B\left(x_{0}, \rho_{0}\right)$.

On en déduit que

$$
E_{x}\left(T_{\rho_{0}}\right) \leqq E_{x}{ }^{a}\left(T_{\rho_{0}}^{a}\right)
$$

De même si $K \geqq b$,

$$
E_{x}\left(T_{\rho_{0}}\right) \geqq E_{x}^{b}\left(T_{\rho_{0}}^{b}\right) .
$$

Les temps moyens de sortie pour les variétés de comparaison ont 
egalement été calculés dans [4]. Si l'on pose

$$
\Phi^{a}(\rho)=\int_{0}^{\rho} \frac{d s}{\theta^{a}(s)} \int_{0}^{s} \theta^{a}(t) d t
$$

On a $\frac{1}{2} E_{x}{ }^{a}\left(T_{\rho_{0}}^{a}\right)=\Phi^{a}\left(\rho_{0}\right)-\Phi^{a}\left(d\left(x_{0}, x\right)\right)$.

Par exemple dans l'espace euclidien de dimension $n$, le temps moyen de sortie partant du centre est:

$$
E_{x_{0}}^{0}\left(T_{\rho_{0}}^{0}\right)=2 \int_{0}^{\rho_{0}} \frac{d s}{s^{n-1}} \int_{0}^{s} t^{n-1} d t=\frac{\rho_{0}^{2}}{n} .
$$

En dimension 2, le temps moyen de sortie partant du centre est, sur la sphère de courbure $+a^{2}$ :

$$
E_{x_{0}}^{a^{2}}\left(T_{\rho_{0}}^{a^{2}}\right)=2 \int_{0}^{\rho_{0}} \frac{a d s}{\sin a s} \int_{0}^{s} \frac{\sin a t}{a} d t=\frac{4}{a^{2}} \log \frac{1}{\cos a \rho_{0} / 2}\left(\rho_{0}<\frac{\pi}{a}\right)
$$

et dans le plan hyperbolique de courbure $-a^{2}$ :

$$
E_{x_{0}}^{-a^{2}}\left(T_{\rho_{0}}^{-a^{2}}\right)=\frac{4}{a^{2}} \log \operatorname{Ch} \frac{a \rho_{0}}{2} .
$$

\section{I耳. Étude de Certaines Classes de Fonctions Harmoniques}

1. Cas de la courbure $\leqq 0$.

Nous allons démontrer très rapidement des résultats de Greene-Wu (voir [8]).

Théorème 4. Soit $V$ une variété riemannienne à courbure $\leqq 0$, simplement connexe. Pour $1 \leqq p<+\infty$, la seule fonction harmonique $L^{p}(V)$ est la fonction identiquenent nulle.

Corollaire. Soit $V$ une variété Kählénienne à courbure $\leqq 0$ simplement connexe. Pour $1 \leqq p<+\infty$, la seule fonction holomorphe $L^{p}(V)$ est la fonction identiquement nulle.

Démonstration du théorème 4. Il suffit comme dans [8] de voir le lemme suivant:

Lemme 3. Soit $V$ une variété à courbure $\leqq 0$, simplement 
connexe, $f$ une fonction $\geqq 0$, sous harmonique. Pour tout $x_{0} \in V$ et tout $r$ tel que $0<r<+\infty$, on $a$ :

$$
f\left(x_{0}\right) \leqq-\frac{1}{\omega_{n-1} r^{n-1}} \int_{S\left(x_{0}, r\right)} f(x) d \sigma_{r}(x)
$$

où $S\left(x_{0}, r\right)$ est la sphère riemannienne de centre $x_{0}$ et de rayon $r$, $d \sigma_{r}$ sa mesure superficielle.

Démonstration du lemme 3. D'après le théorème 3, 1 qui s'applique ici sans restriction sur $r$ on $a$ :

$$
g_{B\left(x_{0}, r\right)}\left(x_{0}, z\right) \leqq g_{B^{0}\left(x_{0}, r\right)}^{0}\left(x_{0}, z\right)
$$

où $g_{B^{0}\left(x_{0}, r\right)}^{0}$ dénote la fonction de Green de la boule euclidienne (identifiée comme toujours à la boule riemannienne). Si $z \in S\left(x_{0}, r\right)$ il résulte que

$$
\frac{\partial g_{B\left(x_{0}, r\right)}}{\partial \vec{n}}\left(x_{0}, z\right) \leqq \frac{\partial g_{B^{0}\left(x_{0}, r\right)}^{0}}{\partial \vec{n}}\left(x_{0}, z\right)
$$

car $g$ est nulle sur la sphère, ainsi que $g^{0}$, et que les longueurs euclidienne et riemannienne radiales sont les mêmes suivant le procédé d'identification des deux boules. Cette inégalité s'écrit encore, avec les noyaux de Poisson:

$$
\Pi_{B\left(x_{0}, r\right)}\left(x_{0}, z\right) \leqq \Pi_{B^{0}\left(x_{0}, r\right)}^{0}\left(x_{0}, z\right)=\frac{1}{\omega_{n-1} r^{n-1}}
$$

On a alors puisque $f$ est sous-harmonique et $\geqq 0$ :

$$
f\left(x_{0}\right) \leqq \int_{S\left(x_{0}, r\right)} \Pi_{B\left(x_{0}, r\right)}\left(x_{0}, z\right) f(z) d \sigma_{r}(z) \leqq \frac{1}{\omega_{n-1} r^{n-1}} \int_{S\left(x_{0}, z\right)} f(z) d \sigma_{r}(z)
$$

comme annoncé.

Le théorème 4 résulte trivialement du lemme 3 appliqué à la fonction $f=|\Psi|^{p}$ où $\Psi$ est une fonction harmonique $L^{p}(V)$, en remarquant que dans ce cas il existe une suite $r_{n} \rightarrow+\infty$ telle que $\int_{S\left(x_{0}, r_{n}\right)}|\Psi(z)|^{p} d \sigma_{r_{n}}(z)$ tende vers 0 .

Remarque. On peut par cette méthode obtenir très facilement d'autres résultats de [8]: par exemple si $K \leqq-a^{2}<0, f \geqq 0$ sous harmonique (pas nécessairement $C^{2}$ ) on a 


$$
\int_{S\left(x_{0}, r\right)} f(z) d_{r}(z) \geqq D(f) e^{\sqrt{n-1} a^{r}}
$$

où $D(f)$ ne dépend que de $f$ et pas de $r$. Cela résulte de l'expression explicite de la fonction de Green globale $g^{-a^{2}}$ et du raisonnement du lemme 3.

2. Cas de la courbure de Ricci $\geqq 0$.

Nous donnons ici un théorème du type de Liouville.

Théorème 5. Soit $V$ une variété riemannienne ayant un rayon d'injectivité global strictement positif, et à courbure de Ricci $\geqq 0$. (1) Les seule fonctions harmoniques bornées sur $V$ sont les constantes.

(2) Les seules fonctions harmoniques à intégrale de Dirichlet finie sont les constantes.

\section{Démonstration.}

(1) Soit $f$ harmonique bornée non constante, et soit $x_{0}$ un point de $V$ tel que $d f\left(x_{0}\right) \neq 0$.

On remarque le temps de vie du brownien sur $V$ est infini par le théorème 2 de [4] (l'hypothèse de courbure dàns ce théorème tel qu'il est énoncé en [4] est que la courbure sectionnelle est minorée, mais il suffit en fait d'une minoration de la courbure de Ricci, car une telle minoration implique le contrôle du $\frac{\theta^{\prime}}{\theta}$ dont on a besoin).

On a alors par la formule de Itô déjà utilisée au théorème 1:

$$
f^{2}\left(X_{t}\right)=f^{2}\left(x_{0}\right)+b_{\tau(t)}+\frac{1}{2} \int_{0}^{t} \Delta f^{2}\left(X_{s}\right) d s
$$

(pour le processus partant de $x_{0}, b_{\tau(t)}$ étant un brownien chargé de temps).

Donc

$$
E_{x_{0}}\left(f^{2}\left(X_{t}\right)\right)=f^{2}\left(x_{0}\right)+\frac{1}{2} \int_{0}^{t} E_{x_{0}}\left[\Delta f^{2}\left(X_{s}\right)\right] d s .
$$

Mais $\Delta f^{2}=2 f . \quad \Delta f+2\|d f\|^{2}=2\|d f\|^{2}$ puisque $f$ est harmonique.

Donc 


$$
E_{x_{0}}\left(f^{2}\left(X_{t}\right)\right)=f^{2}\left(x_{0}\right)+\int_{0}^{t} E_{x_{0}}\left[\|d f\|^{2}\left(X_{s}\right)\right] d s .
$$

Maintenant on a de même

$$
E_{x_{0}}\left[\|d f\|^{2}\left(X_{s}\right)\right]=\left\|d f\left(x_{0}\right)\right\|^{2}+\frac{1}{2} \int_{0}^{t} E_{x_{0}}\left[\Delta\left(\|d f\|^{2}\right)\left(X_{\xi}\right)\right] d \xi
$$

et $\frac{1}{2} \Delta\left(\|d f\|^{2}\right)$ est donné par la formule de Bochner-Lichnérowicz [11], [2]:

$$
\frac{1}{2} \Delta\left(\|d f\|^{2}\right)=\|\operatorname{Hess} f\|^{2}-(d f \mid d \Delta f)+\operatorname{Ric}(d f, d f) .
$$

Ici $\Delta f=0$ et $\operatorname{Ric}(d f, d f) \geqq 0$, d'où

$$
\frac{1}{2} \Delta\left(\|d f\|^{2}\right) \geqq 0 \text {. }
$$

On a donc $E_{x_{0}}\left[\|d f\|^{2}\left(X_{s}\right)\right] \geqq\left\|d f\left(x_{0}\right)\right\|^{2}$ d'où

$$
E_{x_{0}}\left(f^{2}\left(X_{t}\right)\right) \geqq f\left(x_{0}\right)^{2}+\left\|d f\left(x_{0}\right)\right\|^{2} t .
$$

Le second membre tend vers l'infini quant $t$ tend vers l'infini, ce qui est absurde si $f$ est bornée. Donc $d f$ est identiquement nulle, et $f$ est constante.

(2) peut se voir deux façons: soit $f$ une fonction harmonique à intégrale de Dirichlet fini; on se bornera au cas où le brownien de $V$ est transient (le théorème est évident dans le cas où ce brownien est récurrent).

Supposons comme précédemment $f$ non constante et choisissons $x_{0}$ tel que $d f\left(x_{0}\right) \neq 0$. Alors les calculs précédents montrent que

$$
E_{x_{0}}\left[\int_{0}^{+\infty}\|d f\|^{2}\left(X_{s}\right) d s\right]=\int_{V} g_{V}\left(x_{0}, x\right)\|d f(x)\|^{2} d x=+\infty .
$$

En désignant par $U_{\varepsilon}$ l'ensemble des $x \in V$ tels que $g_{V}\left(x_{0}, x\right) \geqq \varepsilon$, on en déduit

$$
\varepsilon \int_{V-U_{\varepsilon}}\|d f(x)\|^{2} d x+\int_{U_{\varepsilon}} g_{V}\left(x_{0}, x\right)\|d f(x)\|^{2} d x=+\infty .
$$

Comme $U_{\varepsilon}$ est relativement compact et $g_{r}$ localement intégrable, on en déduit que

$$
\int_{V-U_{\varepsilon}}\|d f(x)\|^{2} d x=+\infty
$$

pour tout $\varepsilon>0$. Donc a fortiori 


$$
\int_{V}\|d f(x)\|^{2} d x=+\infty
$$

Une autre démonstration consiste à se ramener au (1) au moyen du lemme suivant:

Lemme 4. Soit $V$ une variété riemannienne. S'il existe sur $V$ une fonction harmonique non constante à intégrale de Dirichlet finie, alors il existe aussi sur $V$ une fonction harmonique bornée non bornée non constante.

Démonstration. Soit $F$ la frontière de Martin de $\mathrm{V}, \lambda\left(x_{0}, d y\right)$ la mesure harmonique sur $F$ du brownien de $V$ issu de $x_{0}$. D'après Doob (voir [5]) $f^{2}$ est dominée par une fonction harmonique, donc par le théorèm des martingales $\left\{f\left(X_{t}\right)\right\}$ a une valeur-frontière de $\operatorname{Martin} L^{2}(F, \lambda$ $\left.\left(x_{0}, d y\right)\right)$ et celle-ci est non triviale, donc il existe aussi $g \in L^{\infty}\left(F, \lambda\left(x_{0}\right.\right.$, $d y)$ ) non triviale.

Conjecture. Dans la situation du théorème 6, il n'y a pas de fonctions harmoniques $\geqq 0$ non constantes.

Remarque. Des résultats du même type sont énoncés dans [9] par Greene et Wu.

\section{Propagation de la Chaleur et Géodésiques sur les Variétés a Courbure Negative.}

1. Le processus conditionné et les processus accélérés associés.

Soit $V$ une variété différentiable munie d'un opérateur différentiel du second order à symbole principal défini positif, $\Delta$. Ce symbole principal définit alors sur $V$ une métrique riemannienne, et $\Delta$ coincide avec le laplacien de cette métrique à des termes d'ordre $\leqq 1$ près.

Pour $x_{0} \in V$ et $T>0$ fixés, soit $\Omega_{x_{0}}^{T}(V)$ l'espace des probabilités du processus de diffusion sur $V$ issu de $x_{0}$, de générateur $\frac{1}{2} \Delta$, arrêté au temps $T$. 
L'application $X_{T}: \Omega_{x_{0}}^{T}(V) \rightarrow V$ permet de désintégrer la probabilité $P_{x_{0}}$ : si $\Phi$ est une variable aléatoire $\beta_{T}$-mesurable, et si $A \subset V$ est borélien, alors on a par définition de la probabilité conditionnelle $P_{x_{0}}^{X_{T}=x}$ :

$$
P_{x_{0}}\left(\Phi, 1_{A}\left(X_{T}\right)\right)=\int_{A} P_{x_{0}}^{X_{T}=x}(\Phi) P_{T}\left(x_{0}, x\right) d x
$$

où $P_{t}\left(x_{0}, x\right)$ est le noyau de la chaleur "forward" solution de l'équation d'évolution $\frac{\partial}{\partial t}=\frac{1}{2} \Delta^{*}$ ( $\Delta^{*}$ étant l'adjoint formel de $\Delta$ relativement à la métrique du symbole principal).

La probabilité conditionnelle $P_{x_{0}}^{X_{T}=x}$ est complètement caractérisée par la probabilité cylindrique associée. Une écriture explicite de (1) donne le lemme suivant:

Lemme 5. Soit $0<t_{1}<t_{2}<\cdots<t_{n}<T$ une subdivision quelconque de $[0, T]$, et soint $A_{1}, A_{2}, \cdots, A_{n}$ des boréliens de $V$. On a:

$$
\begin{gathered}
P_{x_{0}}^{X_{X}=y_{0}}\left(X_{t_{1}} \in A_{1}, \cdots, X_{t_{n}} \in A_{n}\right)=\frac{1}{P_{T}\left(x_{0}, y_{0}\right)} \int_{A_{1}} P_{t_{1}}\left(x_{0}, x_{1}\right) d x_{1} \\
\int_{A_{2}} P_{t_{2}-t_{1}}\left(x_{1}, x_{2}\right) d x_{2} \cdots \int_{A_{n}} P_{T-t_{n}}\left(x_{n}, y_{0}\right) d x_{n} .
\end{gathered}
$$

En particulier la densité de probabilité $P_{x_{0}}^{X_{T}=y_{0}}\left(X_{t} \in d x\right)$ est

$$
\Pi_{t}^{T}\left(x_{0}, d x\right)=\frac{P_{t}\left(x_{0}, x\right) P_{T-t}\left(x, y_{0}\right)}{P_{T}\left(x_{0}, y_{0}\right)} d x .
$$

Notations. Posons maintenant $T=\varepsilon$, et soit $\widetilde{X}_{t}^{(\varepsilon)}$ le processus conditionné pour aller de $x_{0}$ à $t=0$ en $y_{0}$ à $t=\varepsilon$. Le processus accéléré associé est $X_{t}^{(\varepsilon)}=\widetilde{X}_{\varepsilon t}^{(\varepsilon)}$ par définition $(0 \leqq t \leqq 1)$. Il va de $x_{0}$ à $t=0$ en $y_{0}$ à $t=1$. Soit $\Omega_{x_{0}}^{y_{0}}(V)$ l'espace des chemins continus paramétrés par $[0,1]$ qui vont de $x_{0}$ à $t=0$ en $y_{0}$ à $t=1$; cet $\Omega_{x_{0}}^{y_{0}}(V)$ peut être pris comme espace de probabilité commun des processus $X_{t}^{(8)}$, les probabilités correspondantes étant alors dénotées $P_{x_{0}}^{y_{0}, \varepsilon}$.

Nous prendrons désormais pour $\Delta$ le laplacien d'une métrique fixée s11r $V$. Nous supposerons que le brownien relatif à cette métrique a un temps de vie infini. 
Théorème 6. Le processus $\left(X_{t}^{(\varepsilon)}, 0 \leqq t \leqq \varepsilon\right)$ conditionné pour partir de $x_{0}$ à $t=0$ et arriver en $y_{0} \grave{a} t=\varepsilon$ est le processus solution de l'équation stochastique inhomogène

$$
\begin{aligned}
\widetilde{X}_{t}{ }^{(\varepsilon) i}=x_{0}{ }^{i} & +\int_{0}^{t} \sigma_{j}{ }^{i}\left(\widetilde{X}_{s}^{(\varepsilon)}\right) d b_{s}^{j}-\frac{1}{2} \int_{0}^{t} g^{k l}\left(\widetilde{X}_{s}^{(\varepsilon)}\right) \Gamma_{k l}^{i}\left(\widetilde{X}_{s}^{(\varepsilon)}\right) d s \\
& +\int_{0}^{t} \frac{\partial}{\partial x_{j}}\left(\log P_{\varepsilon-s}\left(y_{0}, \widetilde{X}_{s}^{(\varepsilon)}\right)\right) g^{i j}\left(\widetilde{X}_{s}{ }^{(\varepsilon)}\right) d s
\end{aligned}
$$

ses probabilités de transition sont $\widetilde{\Pi}_{t}^{\left({ }^{(\varepsilon)}\right.}\left(x_{0}, z\right)$ définies par

$$
\widetilde{\Pi}_{t}^{(\varepsilon)}\left(x_{0}, z\right)=\frac{P_{t}\left(x_{0}, z\right) P_{\varepsilon-t}\left(z, y_{0}\right)}{P_{1}\left(x_{0}, y_{0}\right)}
$$

et son générateur infinitésimal est $\frac{1}{2} \Delta+L_{\nabla \log P_{\varepsilon-t}\left(y_{0}, .\right)}$.

Ici $g_{i j}$ est la matrice de la métrique associée au symbole principal (c'est-à-dire $\left.\Delta=g^{i j}\left(\frac{\partial^{2}}{\partial x^{i} \partial x^{j}}-\Gamma_{i j}^{k} \frac{\partial}{\partial x^{k}}\right)\right), \sigma_{j}^{i}$ est la matrice racine carrée symétrique de la matrice inverse $g^{i j}$ (c'est-à-dire $\sum_{k=1}^{n} \sigma_{k}{ }^{i} \sigma_{j}{ }^{k}=g^{i j}$ ), $\nabla$ désigne le gradientau sens de la métrique riemannienne $\left(c^{k=1}\right.$ est-à-dire $\left.\nabla^{i}=g^{i j} \frac{\partial}{\partial x^{j}}\right)$, et $L$ désigne la dérivée de Lie selon un champ de vecteurs; enfin $b^{j}$ est un brownien euclidien sur $R^{n}$.

Démonstration du théorème 6. Considérons la variété $V \times \mathbb{R}^{+}$(la coordonné suivant $R^{+}$étant notée $t$ ), munie de l'opérateur $\frac{1}{2} \Delta+\frac{\partial}{\partial t}$. La diffusion associée est alors le processus de la chaleur de Doob: partant de $\left(x_{0}, t_{0}\right)$ ce processus est décrit comme $Y_{t}=\left(X_{t}^{x_{0}}, t_{0}+t\right)$ où $X_{t}^{x_{0}}$ est le brownien riemannien issu à $t=0$ de $x_{0}$, de générateur $\frac{1}{2} \Delta$; les fonctions harmoniques sont alors les solutions de $\frac{1}{2} \Delta+\frac{\partial}{\partial t}=0$.

Posons $h(z, t)=P_{\varepsilon-t}\left(z, y_{0}\right)$. C'est la fonction de Green de l'équation de la chaleur $\frac{1}{2} \Delta+\frac{\partial}{\partial t}$ de pôle $\left(y_{0}, \varepsilon\right)$; par suite d'après Doob (voir [6]) le h-processus de la chaleur $Y_{t}^{(h)}$ partant de $\left(x_{0}, 0\right) \in V \times \mathbb{R}^{+}$aboutit presque sûrement à $\left(y_{0}, \varepsilon\right)$ et a pour transitions

$$
P_{\left(x_{0}, s\right)}^{(h)}\left(Y_{t}^{(h)} \in d x \otimes d t\right)=\left(P_{t}\left(x_{0}, d x\right) \otimes \delta_{t}(d t)\right) \frac{h(x, t)}{h\left(x_{0}, 0\right)} .
$$

où $\delta_{t}(d t)$ est la masse de Dirac en $t \in \mathbb{R}^{+}$; la projection $X_{t}^{(h)}$ de ce 
processus sur $V$ est un Markov ayant les transitions $\widetilde{\Pi}_{t}^{(\varepsilon)}\left(x_{0}, d x\right)$ $=\frac{P_{t}\left(x_{0}, d x\right) P_{\varepsilon-t}\left(x, y_{0}\right)}{P_{\varepsilon}\left(x_{0}, y_{0}\right)}$, donc $X_{t}^{(h)}$ n'est autre, d'après le lemme 5 , que $\widetilde{X}_{t}{ }^{(\varepsilon)}$.

Le générateur infinitésimal de $Y_{t}^{(h)}$ est défini par

$$
g \Psi(x, t)=\frac{1}{h(x, t)}\left(\frac{1}{2} \Delta_{x}+\frac{\partial}{\partial t}\right)(h(x, t) \Psi(x, t))
$$

de sorte que si l'on prend $\Psi$ indépendante de $t$ et en tenant compte de $\left(\frac{1}{2} \Delta+\frac{\partial}{\partial t}\right) h(x, t)=0$ il vient

$$
\widetilde{g}^{(\varepsilon)} \Psi=\frac{1}{2} \Delta \Psi+L_{\nabla_{x} \log h(x, t)} \Psi=\frac{1}{2} \Delta \Psi+L_{\nabla_{x} \log P_{\varepsilon-t}\left(y_{0}, x\right)} \Psi .
$$

On a bien pour le processus $\widetilde{X}_{t}{ }^{(\varepsilon)}$ le générateur infinitésimal annoncé. Le fait que ce processus soit solution de l'équation stochastique indiquée résulte alors des propriétés classique des diffusions.

2. Le cas simplement connexe à courbure $\leqq 0$ bornée.

Lemme 6. Soient $x, y$ deux points de la variété standard à courbure négative $-a^{2}$. On $a$ :

$$
\lim _{t \rightarrow 0}(2 \pi t)^{n / 2} e^{d^{2}(x, y) / 2 t} P_{t}(x, y)=\left(\frac{a d(x, y)}{\operatorname{Sh}(a d(x, y))}\right)
$$

(étant entendu que le second membre vaut 1 si $x=y$ ).

Démonstration. On note $\theta(x, y)$ la fonction densité de volume riemannien en coordonnées cartésiennes exponentielles de centre $x$. On considère les fonctions $\iota_{i}$ de McKean-Singer-Minakshisundaram-Pleijel:

$$
\begin{aligned}
u_{0}(x, y)= & \theta^{-1 / 2}(x, y) \\
u_{i}(x, y)=\frac{1}{2} & \theta^{-1 / 2}(x, y) \int_{0}^{1} \theta^{1 / 2}\left(x, \exp _{x}\left(\tau \exp _{x}{ }^{-1}(y)\right)\right) \\
& \quad \times \Delta_{y} u_{i-1}\left(x, \exp _{x}\left(\tau \exp _{x}^{-1}(y)\right)\right) \tau^{i-1} d \tau
\end{aligned}
$$

(cf. [2], [3]).

On sait que si l'on pose

$$
S_{k}(t ; x, y)=(2 \pi t)^{-n / 2} e^{-d^{2}(x, y) / 2 t} \sum_{i=0}^{k} t^{i} u_{i}(x, y)
$$


on a:

$$
\frac{\partial S_{k}}{\partial t}(t ; x, y)=\frac{1}{2} \Delta_{y} S_{k}(t ; x, y)+(2 \pi)^{-n / 2} \frac{t^{k-(n / 2)}}{2^{k+1}} e^{-d^{2}(x, y) / 2 t} \Delta_{u} u_{k}(x, y) .
$$

Dans le cas de $V^{-a^{2}}$ les $u_{i}$ ne dépendent que de $r=d(x, y)$ et sont données par

$$
\begin{gathered}
u_{0}(r)=\left(\frac{a r}{\text { Sh } a r}\right)^{(n-1) / 2} \\
u_{i}(r)=\frac{1}{2}\left(\frac{a r}{\operatorname{Sh} a r}\right)^{(n-1) / 2} \int_{0}^{1}\left(\frac{\operatorname{Sh} a r}{a r}\right)^{(n-1) / 2}\left(u_{i-1}^{\prime \prime}(\tau r)\right. \\
\left.+(n-1) \operatorname{coth}(a \tau r) u_{i-1}^{\prime}(\tau r)\right) \tau^{i-1} d \tau .
\end{gathered}
$$

Colin de Verdière [3] a introduit la condition (E) suivante, susceptible d'être vérifiée ou non par la variété $V$ simplement connexe à courbure $\leqq 0$ :

Condition ( $\mathbb{E})$. Il existe des constantes $C$ et $h$ telles que pour tout $i \leqq n$ on ait pour tous $x, y \in V$

$$
\begin{aligned}
& \left|u_{i}(x, y)\right| \leqq C e^{h d(x, y)} \\
& \left|\Delta u_{i}(x, y)\right| \leqq C e^{h d(x, y)}
\end{aligned}
$$

Le même auteur établit alors que si la condition (E) est satisfaite, pour tout $\xi_{0}>0$ il existe une constante $C^{\prime}\left(\xi_{0}\right)$ telle que l'on ait

$$
\left|P_{t}(x, y)-S_{[n-1 / 2]}(t ; x, y)\right| \leqq C^{\prime}\left(\xi_{0}\right) e^{h d(x, y)} \exp \left(-\frac{\xi_{0}}{2} d^{2}(x, y)\right)
$$

pour tous $x, y \in V$ et tout $t$ tel que $0<t \leqq \frac{1}{\xi_{0}}$.

Qui plus est, un examen attentif des estimations de [3], § I. 1, qui conduisent au contrôle précédent, montre que $C^{\prime}\left(\xi_{0}\right)$ reste borné lorsque $\xi_{0}$ reste supérieur à une constante strictement positive. On en conclut qu'il existe une constante $C^{\prime \prime}$ telle que l'on ait

$$
\left|P_{t}(x, y)-S_{[n-1 / 2]}(t ; x, y)\right| \leqq C^{\prime \prime} e^{h d(x, y)} e^{-d^{2}(x, y) / 2 t}
$$

pour tous $x, y \in V$ et tout $t$ tel que $0<t \leqq 1$.

Il est alors clair que le lemme 6 sera établi si nous pouvons montrer que la variété standard $V^{-a^{2}}$ satisfait la condition (E). 
Pour cela nous procédons comme suit: appelons "fonction acceptable" toute fonction analytique sur $V$ qui peut s'exprimer comme une fraction rationnelle en les puissances entières et demi-entières de $r$, Sh ar et Ch ar. Il est clair qu'une fonction acceptable adment une majorante de la forme $C e^{h r}$. On remarque aussi que les dérivées successives par rapport à $r$ d'une fonction acceptable sont des fonctions acceptables.

$\mathrm{Au}$ moyen d'intégrations par parties on peut mettre les $u_{i}$ sous la forme

$$
u_{i}(r)=C_{i}+F_{i}(r) u_{i-1}(r)+G_{i}(r) u_{i-1}^{\prime}(r)+\int_{0}^{1} H_{i}(\tau r, \tau) u_{i-1}(\tau r) d \tau
$$

où $C_{i}$ est une constante, $F_{i}(r)$ et $G_{i}(r)$ des fonctions acceptables, et $H_{i}(\rho, \tau)$ un polynôme en $\tau$ à coefficients fonctions acceptables de $\rho$.

On a des expressions analogues pour les dérivées radiales de $u_{i}$ au moyen des dérivées radiales de $u_{i-1}$ et de fonctions acceptables.

Ces expressions montrent que si $u_{i-1}$ et ses dérivées satisfont des majorations en $C e^{h r}$ il en sera de même de $u_{i}$ et de ses dérivées. Or cette propriété est vraie pour $u_{0}$ qui est une fonction acceptable. Donc la propriété est vraie pour les $u_{i}$. Comme la condition (E) ne fait intervenir qu'un nombre fini des $u_{i}$ et leurs laplaciens, le lemme est établi.

Remarque. On peut vérifier grâce à la formule de récurrence de Millson (voir remarque 2 après le théorème 1 ) qu'en dimension impaire les $u_{i}$ sont en fait des fonctions acceptables (et même des fractions rationnelles en $r$, Ch $a r$, Sh $a r$ ). Ce n'est pas vrai en dimension paire.

Théorème 7. Soit $V$ simplement connexe à courbure $\leqq 0$ bornée, $x_{0}$ et $y_{0}$ deux points de $V$, et $\left(X_{t}^{(\varepsilon)}, t \in[0,1]\right)$ le processus conditionné accéléré correspondant. Alors, quand $\varepsilon$ tend vers 0 , pour tout $t \in[0$, 1] la loi de la variable aléatoire $X_{t}^{\left({ }^{(\varepsilon)}\right.}$ converge étroitement vers la mesure de Dirac $\delta_{r(t)}$, où $\gamma$ est l'unique géodésique minimisante joignant $x_{0}$ à $y_{0}$, paramétrée par $[0,1]$ proportionnellement à l'abscisse curviligne.

C'est évident pour $t=0$ et $t=1$. Pour $t \in] 0,1$ [ il suffit de montrer que pour toute fonction $\Psi$ continue bornée sur $V$, nulle au point $\gamma(t)$, 
on a:

$$
\lim _{\varepsilon \rightarrow 0} \int_{V} \Pi_{t}^{(\varepsilon)}\left(x_{0}, z\right) \Psi(z) d z=0 .
$$

On peut supposer $\Psi \geqq 0$. Soit $\eta>0$. Considérons une boule de centre $r(t)$ et de rayon $a>0$. On peut écrire:

$$
\begin{aligned}
\int_{V} \Pi_{t}^{(\varepsilon)}\left(x_{0}, z\right) \Psi(z) d z= & \int_{B(r(t), a)} \Pi_{t}{ }^{(\varepsilon)}\left(x_{0}, z\right) \Psi(z) d z \\
& +\int_{V-B(r(t), a)} \Pi_{t}^{(\varepsilon)}\left(x_{0}, z\right) \Psi(z) d z
\end{aligned}
$$

En prenant a assez petit, $\Psi(z)$ sera majoré par $\frac{\eta}{2}$ sur la boule, et la première intégrale sera plus petite que $\frac{\eta}{2}$. Fixons donc un tel $a$. Il reste à voir que la seconde intégral tend vers 0 quand $\varepsilon \rightarrow 0$.

Choisissons $b>0$ tel que l'on ait $-b^{2} \leqq K \leqq 0$. On a alors par le théorème 1 :

$$
P_{t}^{\left(-b^{2}\right)}\left(x_{0}, z\right) \leqq P_{t}\left(x_{0}, z\right) \leqq P_{t}^{(0)}\left(x_{0}, z\right) .
$$

En faisant le changement de variable $t \rightarrow \varepsilon t$ dans les $\widetilde{\Pi}_{l}^{(\varepsilon)}$ donnés au théorème 6 il vient

$$
\Pi_{t}^{(\varepsilon)}\left(x_{0}, z\right)=\frac{P_{\varepsilon t}\left(x_{0}, z\right) P_{\varepsilon(1-t)}\left(z, y_{0}\right)}{P_{\varepsilon}\left(x_{0}, y_{0}\right)}
$$

d'où

$$
\Pi_{\iota}^{(\varepsilon)}\left(x_{0}, z\right) \leqq \frac{P_{\varepsilon t}^{(0)}\left(x_{0}, z\right) P_{\varepsilon(1-t)}^{(0)}\left(z, y_{0}\right)}{P_{\varepsilon}^{\left(-b^{2}\right)}\left(x_{0}, y_{0}\right)}
$$

Nous aurons donc

$$
\begin{aligned}
& \int_{V-B(r(t), a)} I t^{(\varepsilon)}\left(x_{0}, z\right) \Psi(z) d z \frac{(2 \pi \varepsilon)^{n / 2} e^{d^{2}\left(x_{0}, y_{0}\right) / 2 \varepsilon}}{(2 \pi \varepsilon)^{n / 2} e^{d^{2}\left(x_{0}, y_{0}\right) / 2 \varepsilon} P_{\iota}^{\left(-b^{2}\right)}}\left(x_{0}, y_{0}\right) \\
& \times \int_{V-B(\gamma(t), a)} \frac{e^{-d^{2}\left(x_{0}, z\right) / 2 \varepsilon t}}{(2 \pi \varepsilon t)^{n / 2}} \frac{e^{-d^{2}\left(z, y^{0}\right) / 2 \varepsilon(1-t)}}{(2 \pi \varepsilon(1-t))^{n / 2}} \Psi(z) d z .
\end{aligned}
$$

D'après le lemme $6,(2 \pi \varepsilon)^{n / 2} e^{d^{2}\left(x_{0}, y_{0}\right) /(2 \varepsilon)} P_{t}^{\left(-b^{2}\right)}\left(x_{0}, y_{0}\right)$ a une limite $>0$. Il suffit donc de voir que:

$$
\lim _{\varepsilon \rightarrow 0} \int_{V-B(r(t), a)} \frac{\exp \left(\frac{d^{2}\left(x_{0}, y_{0}\right)}{2 \varepsilon}-\frac{d^{2}\left(x_{0}, z\right)}{2 \varepsilon t}-\frac{d^{2}\left(z, y_{0}\right)}{2 \varepsilon(1-t)}\right)}{(2 \pi \varepsilon t(1-t))^{n / 2}} \Psi(z) d z=0 .
$$


Pour contrôler l'exponentielle, résolvons dans $V$, à courbure $\leqq 0$, les deux triangles $x_{0} z \gamma(t)$ et $y_{0} z \gamma(t)$ :

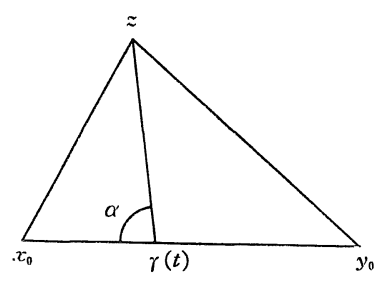

Par comparaison avec l'espace euclidien, on a:

$$
\begin{aligned}
& d^{2}\left(x_{0}, z\right) \geqq d^{2}\left(x_{0}, \gamma(t)\right)+d^{2}(\gamma(t), z)-2 d\left(x_{0}, \gamma(t)\right) d(\gamma(t), z) \cos \alpha \\
& d^{2}\left(z, y_{0}\right) \geqq d^{2}\left(y_{0}, \gamma(t)\right)+d^{2}(\gamma(t), z)+2 d\left(x_{0}, \gamma(t)\right) d(\gamma(t), z) \cos \alpha .
\end{aligned}
$$

En tenant compte de ce que $d\left(x_{0}, \gamma(t)\right)=t d\left(x_{0}, y_{0}\right)$ et $d\left(y_{0}, \gamma(t)\right)=(1-t)$ $d\left(x_{0}, y_{0}\right)$, on en déduit:

$$
\frac{1}{t} d^{2}\left(x_{0}, z\right)+\frac{1}{1-t} d^{2}\left(z, y_{0}\right) \geqq d^{2}\left(x_{0}, y_{0}\right)+\frac{1}{t(1-t)} d^{2}(\gamma(t), z) .
$$

Il en résulte que l'exponentielle est majorée par $\exp \left(-\frac{1}{2 t(1-t)} d^{2}(\gamma(t), z)\right)$.

Finalement

$$
\int_{V-B(r(t), a)} \Pi_{t}^{(\varepsilon)}\left(x_{0}, z\right) \Psi(z) d z \leqq Q(\varepsilon) \int_{V-B(r(t), a)} \frac{e^{-d^{2}(r(t), z) / 2 \varepsilon t(1-t)}}{(2 \pi \varepsilon t(1-t))^{n / 2}} \Psi(z) d z
$$

avec $\lim _{\varepsilon \rightarrow 0} Q(\varepsilon)<+\infty$.

La dernière intégrale est majorée par:

$$
\omega_{n-1}\|\Psi\| \int_{a}^{+\omega} \frac{e^{-\rho^{2} / 2 \varepsilon t(1-t)}}{(2 \pi \varepsilon t(1-t))^{n / 2}}(\operatorname{Sh} a \rho)^{n-1} d \rho .
$$

Elle tend vers 0 , ce qui établit le théorème.

\section{A-t-on convergence presque sure vers la géodésique?}

On peut se demander si pour presque tout $(1)$ la trajectoire $\left(X_{t}{ }^{(\varepsilon)}\right.$ $(\omega))_{t \in[0,1]}$ coverge vers la géodésique $\gamma$.

Pour discuter ce point, nous procéderons comme suit: Nous avons $\mathrm{vu}$ (théorème 6) que le processus conditionné pour aller de $x_{0}$ à $y_{0}$ dans 
le temps $\varepsilon$, noté $\left(\widetilde{X}_{t}{ }^{(\varepsilon)}, t \in[0, \varepsilon]\right)$, est solution de l'équation stochastique

$$
\begin{aligned}
\widetilde{X}_{t}{ }^{(\varepsilon) i}=x_{0}{ }^{i} & +\int_{0}^{t} \sigma_{j}{ }^{i}\left(\widetilde{X}_{t}^{(\varepsilon)}\right) d b_{s}{ }^{j}-\frac{1}{2} \int_{0}^{t} g^{k l}\left(\widetilde{X}_{s}{ }^{(\varepsilon)}\right) \Gamma_{k l}^{i}\left(\widetilde{X}_{s}^{(\varepsilon)}\right) d s \\
& +\int_{0}^{t} g^{i j}\left(\widetilde{X}_{s}^{(\varepsilon)}\right) \frac{\partial}{\partial x_{j}}\left(\log P_{\varepsilon-s}\left(y_{0}, \widetilde{X}_{s}{ }^{(\varepsilon)}\right)\right) d s .
\end{aligned}
$$

Les coefficients de cette équation ne sont pas bornés sur $\mathbb{R}^{n}$ et il sera avantageux de nous ramener au cas où certains le sont: pour cela soit $V$ un voisinage compact de la géodésique $\gamma$, et soit $\alpha$ une fonction $C^{1}$ sur $V$ à valeurs $[0,1]$, égale à 1 sur $V$ et nulle au voisinage de l'infini. Posons:

$$
\begin{aligned}
& \underline{g}^{k l}(x)=\alpha(x) g^{k l}(x) \\
& \underline{\sigma}_{j}^{i}(x)=\alpha(x) \sigma_{j}^{i}(x) .
\end{aligned}
$$

Alors les $\underline{g}^{k l}$ et les $\underline{\sigma}_{j}^{i}$ sont nuls au voisinage de l'infini.

La propriété de localisation des équations stochastiques nous dit que si $T^{(\varepsilon)}(\omega)$ est le premier instant où $\widetilde{X}_{t}^{(\varepsilon)}(\omega)$ sort de $V$, on a presque sûrement pour $t<T^{(\varepsilon)}(\omega)$

$$
\begin{aligned}
\widetilde{X}_{t}^{(\varepsilon) i}=x_{0}{ }^{i} & +\int_{0}^{t} \underline{\sigma}_{j}{ }^{i}\left(\widetilde{X}_{s}{ }^{(\varepsilon)}\right) d b_{s}{ }^{j}-\frac{1}{2} \int_{0}^{t} \underline{g}^{k l}\left(\widetilde{X}_{s}{ }^{(\varepsilon)}\right) \Gamma_{k l}^{i}\left(\widetilde{X}_{s}{ }^{(\varepsilon)}\right) d s \\
& +\int_{0}^{t} \underline{g}^{i j}\left(\widetilde{X}_{s}{ }^{(\varepsilon)}\right) \frac{\partial}{\partial x^{j}}\left(\log P_{\varepsilon-s}\left(y_{0}, \widetilde{X}_{s}{ }^{(\varepsilon)}\right)\right) d s .
\end{aligned}
$$

Mais le "presque sûrement" dépend de $\varepsilon$. Pour éviter cette difficulté, nous considérerons, plutôt que le paramètre continu $\varepsilon$, des suites $\left\{\varepsilon_{\nu}\right\}_{\nu \in \mathbb{N}}$, convergeant vers 0 .

Une tells suite étant choisie, nous pouvons dire que presque sûrement on a pour tout $\nu$ :

$$
\begin{aligned}
\widetilde{X}_{t}^{(\varepsilon) i}=x_{0}{ }^{i} & +\int_{0}^{t} \underline{\sigma}_{j}^{i}\left(\widetilde{X}_{s}^{\left(\varepsilon_{\nu}\right)} d b_{s}^{j}-\frac{1}{2} \int \underline{g}^{k l}\left(\widetilde{X}_{s}{ }^{\left(\varepsilon_{\nu}\right)}\right) \Gamma_{k l}^{i}\left(\widetilde{X}_{s}^{\left(\varepsilon_{\nu}\right)}\right) d s\right. \\
& +\int_{0}^{t} \underline{g}^{i j}\left(\widetilde{X}_{s}^{\left(\varepsilon_{\nu}\right)}\right) \frac{\partial}{\partial x_{j}}\left(\log P_{\varepsilon-s}\left(y_{0}, \widetilde{X}_{s}{ }^{\left(\varepsilon_{\nu}\right)}\right)\right) d s
\end{aligned}
$$

pour $t \in\left[0, T^{\left(\varepsilon_{\nu}\right)}(\omega)\right]$.

Si l'on veut établir la convergence uniforme de $X_{t}^{\left(\varepsilon_{\nu}\right)}$ vers la géodésique, il suffit de raisonner sur le processus déduit de la solution de (2) par le changement de temps $t \rightarrow \varepsilon t$. En effet, si l'on a démontré que ce 
processus converge presque sûrement uniformément vers $\gamma$, il en résulte que presque sûrement ce processus reste dans $V$ pour assez grand. Il coincide alors avec $X_{t}^{\left(\varepsilon_{\nu}\right)}$, d'où la convergence de $X_{t}^{\left(\varepsilon_{\nu}\right)}$ vers $\gamma$.

Désormais donc nous désignerons par $X_{t}{ }^{\left(\varepsilon_{\nu}\right)}$ la solution changée de temps de l'équation (2), et non plus de l'équation (1).

Nous avons

$$
\begin{aligned}
X_{t}^{\left(\varepsilon_{\nu}\right)}=x_{0}{ }^{i} & +\int_{0}^{\varepsilon_{\nu} t} \underline{\sigma}_{j}^{i}\left(\widetilde{X}_{s}^{\left(\varepsilon_{\nu}\right)}\right) d b_{s}^{j}-\frac{1}{2} \int_{0}^{\varepsilon_{\nu} t} \underline{g}^{k l}\left(\widetilde{X}_{s}^{\left(\varepsilon_{\nu}\right)}\right) \Gamma_{k l}^{i}\left(\widetilde{X}_{s}^{\left(\varepsilon_{\nu}\right)}\right) d s \\
& +\int_{0}^{\varepsilon_{\nu} t} \underline{g}^{i j}\left(\widetilde{X}_{s}{ }^{\left(\varepsilon_{\nu}\right)}\right) \frac{\partial}{\partial x^{j}}\left(\log P_{\varepsilon_{\nu}-s}\left(y_{0}, \widetilde{X}_{s}{ }^{\left(\varepsilon_{\nu}\right)}\right)\right) d s .
\end{aligned}
$$

La seconde intégrale est majorée par $C^{t e} \times \varepsilon_{\nu}$, donc tend vers 0 uniformémént pour $t \in[0,1]$ quand $\varepsilon_{\nu} \rightarrow 0$.

La première s'écrit $\tilde{b}_{\tilde{\omega}_{\nu}}\left(\tau\left(\varepsilon_{\nu} t\right)\right)$ où $\tilde{b}$ est un brownien standard et $\omega \rightarrow \widetilde{\omega}_{\nu}$ un isomorphisme (dépendant de $\nu$ ) d'espaces de probabilité, tandis que le changement de temps $\tau$ est donné par

$$
\tau\left(\varepsilon_{\nu}\right)=\int_{0}^{\varepsilon_{\nu} t} \sum_{j=1}^{n}\left|\underline{\sigma}_{j}^{i}\left(\widetilde{X}_{s}^{\left(\varepsilon_{\nu}\right)}\right)\right|^{2} d s=\int_{0}^{\varepsilon_{\nu} t} \underline{g}^{i i}\left(\widetilde{X}_{s}^{\left(\varepsilon_{\nu}\right)}\right) d s .
$$

On voit que $\tau\left(\varepsilon_{\nu} t\right) \leqq A \varepsilon_{\nu} t$ pour une certaine constante $\mathrm{A}$. Puisque $\tilde{b}$ est un brownien standard on a pour tout $\nu$

$$
P\left\{\sup _{0 \leqq s \leqq A \varepsilon_{\nu}}\left|\tilde{b}_{\tilde{\omega}_{\nu}}(s)\right|>\frac{1}{\nu}\right\} \leqq \nu^{2} E\left[\left(b_{\tilde{\omega}_{\nu}}\left(A \varepsilon_{\nu}\right)\right)^{2}\right] \leqq A \nu^{2} \varepsilon_{\nu}
$$

d'où a fortiori

$$
P\left\{\sup _{0 \leqq t \leqq 1}\left|\hat{b}_{\hat{\omega}_{\nu}}\left(\tau\left(\varepsilon_{\nu}\right)\right)\right|>\frac{1}{\nu}\right\} \leqq A \nu^{2} \varepsilon_{\nu}
$$

Si la série $\sum_{\nu} \nu^{2} \varepsilon_{\nu}$ converge, on a presque sûrement pour $\nu$ asscz grand

$$
\sup _{0 \leqq t \leqq 1}\left|\tilde{b}_{\tilde{\omega}_{\nu}}\left(\tau\left(\varepsilon_{\nu} t\right)\right)\right| \leqq \frac{1}{\nu} .
$$

On conclut de là que pour des suites $\hat{\varepsilon}_{\nu}$ convergeant assez vite vers 0 la première intégrale tend vers 0 uniformément en $t \in[0,1]$.

Dans la suite nous nous bornerons donc à des suites $\varepsilon_{\nu}$ convergeant asse $z$ vite vers 0 pour que cela ait lieu.

Pour traiter la troisième intégrale nous faisons le changement de variable $s=\varepsilon_{\nu} \xi$. 


$$
\begin{aligned}
\int_{0}^{\varepsilon_{\nu} t} & \underline{g}^{i j}\left(\widetilde{X}_{s}{ }^{\left(\varepsilon_{\nu}\right)}\right) \frac{\partial}{\partial x^{j}}\left(\log P_{\varepsilon_{\nu}-s}\left(y_{0}, \widetilde{X}_{s}^{\left({ }^{\left(\varepsilon_{\nu}\right)}\right)}\right) d s\right. \\
\quad= & \varepsilon_{\nu} \int_{0}^{t} \underline{g}^{i j}\left(X_{\xi}{ }^{\left(\varepsilon_{\nu}\right)}\right) \frac{\partial}{\partial x^{j}}\left(\log P_{\varepsilon_{\nu}(1-\xi)}\left(y_{0}, X_{\xi}{ }^{\left(\varepsilon_{\nu}\right)}\right)\right) d \xi .
\end{aligned}
$$

Posons $\quad P_{t}\left(y_{0}, x\right)=\frac{e^{-d^{2}\left(y_{0}, x\right) / 2 t}}{(2 \pi t)^{n / 2}} U_{t}\left(y_{0}, x\right)$

Alors on a dans la carte exponentielle d'origine $y_{0}$ :

$$
\begin{aligned}
\frac{\partial}{\partial x^{j}}\left(\log P_{\varepsilon_{\nu}(1-\xi)}\left(y_{0}, x\right)\right) & =-\frac{1}{\varepsilon_{\nu}(1-\xi)} g_{j k}\left(y_{0}\right) x^{k} \\
& +\frac{1}{U_{\varepsilon_{\nu}(1-\xi)}\left(y_{0}, x\right)} \frac{\partial}{\partial x^{j}}\left(U_{\varepsilon_{\nu}(1-\xi)}\left(y_{0}, x\right)\right)
\end{aligned}
$$

Alors la troisième intégrale devient

$$
\begin{aligned}
-\int_{0}^{t} F^{i}\left(\xi, X_{\xi}^{\left(\varepsilon_{\nu}\right)}\right) d \xi+\varepsilon_{\nu} \int_{0}^{t} & \frac{g^{i j}\left(X_{\xi}^{\left(\varepsilon_{\nu}\right)}\right)}{U_{\varepsilon_{\nu}(1-\xi)}\left(y_{0}, X_{\xi}^{\left(\varepsilon_{\nu}\right)}\right)} \frac{\partial}{\partial x^{i}} \\
& \times\left(U_{\varepsilon_{\nu}(1-\xi)}\left(y_{0}, X_{\xi}^{\left(\varepsilon_{\nu}\right)}\right)\right) d \xi
\end{aligned}
$$

où l'on posé

$$
F^{i}(\xi, x)=\frac{1}{1-\xi} \underline{g}^{i j}(x) g_{j k}\left(y_{0}\right) x^{k}
$$

(On observera ici que, la fonction plateau $\alpha$ ayant été choisie suffisamment régulière, $F_{i},(\xi, x)$ est lipschitzienne en $x$, avec une constante de Leipschitz uniforme pour $\xi \in\left[0, t_{0}\right]$, quel que soit $\left.t_{0}<1\right)$.

Regardons le second terme de (A). On note que $U_{\varepsilon_{y}(1-\xi)}\left(y_{0}, x\right)$ est minoré par $U_{\varepsilon_{y}(1-\xi)}^{\left(-b^{2}\right)}\left(y_{0}, x\right)$. D'après le lemme 6 , cette dernière fonction converge pour $\varepsilon_{\nu} \rightarrow 0$ vers une quantité strictement positive. La convergence est évidemment uniforme pour $\xi \in[0,1]$ et par ailleurs la démonstration du lemme 6 fait voir qu'elle est uniforme en $x$ lorsque $x$ reste dans un compact. Comme $\underline{g}^{i j}(x)$ est nul hors d'un compact, on conclut que les quantités $\frac{g^{i j}(x)}{U_{\varepsilon_{\nu}(1-\xi)}\left(y_{0}, x\right)}$ sont uniformément bornées pour $\xi \in[0,1], x$ $\in V$ et $\varepsilon_{\nu} \rightarrow 0$.

Supposons alors que la condition suivante soit satisfaite (par la variété $V):$

Condition (C). Pour tout compact $K$ de $V \times V$, il existe $t_{0}>0$ 
tel que $V_{y} U_{t}(x, y)$ soit uniformément borné sur $\left.] 0, t_{0}\right] \times K$.

Sous cette condition, nous voyons que l'intégrande du second terme de (A) est borné uniformément pour $\varepsilon_{\nu} \rightarrow 0$. Donc ce terme tend vers 0 , uniformément en $t \in[0,1]$.

On a donc établi que presque sûrement (pour des suites $\varepsilon_{\nu}$ tendant vers 0 assez vite)

$$
\lim _{\varepsilon_{\nu} \rightarrow 0}\left(X_{t}^{\left(\varepsilon_{\nu}\right) i}-x_{0}^{i}+\int_{0}^{t} F^{i}\left(\xi, X_{\xi}^{\left(\varepsilon_{\nu}\right)}\right) d \xi\right)=0
$$

uniformément pour $t \in[0,1]$.

Alors, la condition de Lipschitz que satisfait $F(\xi, x)$, uniforme pour $\xi \in\left[0, t_{0}\right]$ pour tout $t_{0}<1$, entraîne par des estimations classiques en théorie des équations différentielles que presque sûrement $X_{t}^{\left(\varepsilon_{\nu}\right)}$ converge, uniformément sur $\left[0, t_{0}\right]$ pour tout $t_{0}<1$, vers la solution du problème de Cauchy

$$
\left\{\begin{array}{l}
\frac{d Y^{i}}{d \xi}=-F_{i}\left(\xi, Y^{i}\right) \\
Y^{i}(0)=x_{0}^{i}
\end{array}\right.
$$

et il est aisé de vérifier (en se souvenant qu'on est dans la carte exponentielle d'origine $x_{0}$ ) que la solution de ce problème de Cauchy est

$$
\gamma^{i}(\xi)=(1-\xi) x_{0}^{i}
$$

c'est-à-dire précisément la géodésique $\gamma$.

On peut donc conclure par la proposition suivante:

Proposition. Si la variété $V$, simplement connexe à courburc négative bornée, satisfait de plus la condition (C), alors pour toute suite $\left\{\varepsilon_{\nu}\right\}_{\nu \in \mathbf{N}}$ de réels $>0$, convergeant assez vite vers 0 , le processus conditionné accéléré $\left(X_{t}{ }^{\left(\varepsilon_{\nu}\right)}, t \in[0,1]\right)$ converge puisque sûrement vors la géodésique $\gamma$, uniformément pour $t \in\left[0, t_{0}\right]$ quel que soit $t_{0}<1$.

La condition (C) que nous avons introduite n'est pas snas rapport avec la condition (E) de Colin de Verdière [3], déjà utilisée au lemme 6. Il est clair que la condition (E) sera satisfaite si la différence

$$
P_{t}(x, y)-S_{[(n-1) / 2]}(t, x, y)
$$


est suffisamment bien contrôlée non seulement au sens $C^{0}$, mais encore au sens $C^{1}$, plus précisément si l'on a une estimation de la forme

$$
\left\|\nabla_{y} P_{t}(x, y)-\nabla_{y} S_{[(n-1) / 2]}(t ; x, y)\right\| \leqq C^{\prime}\left(\xi_{0}\right) e^{h d(x, y)} e^{-\frac{\xi_{0}}{2} d^{2}(x, y)}\left(0<t \leqq \frac{1}{\xi_{0}}\right)
$$

avec $C^{\prime}\left(\xi_{0}\right)$ bornée pour $\xi_{0} \geqq C^{t e}>0$.

Cela est réalisé si des estimées en $C e^{h r}$ sont satisfaites non seulement par les $u_{i}$ et leurs laplaciens, mais encore par les gradients de ces fonctions. C'est ainsi qu'en utilisant les résultats de [3], §.1.3, on peut montrer que la condition (C) est satisfaite en dimension 2.

D'autre part, les expressions des $u_{i}$ invoquées dans la démonstration du lemme 6 permettent de montrer que la condition (C) est satisfaite en courbure constante négative.

\section{Le cas d'une variété compacte à courbure négative.}

Notations. Soit $V$ une variété riemannienne compacte à courbure négative, et soit $\pi: \widetilde{V} \rightarrow V$ son revêtement universel, de sorte que $\widetilde{V}$ est simplement connexe à courbure négative bornée $\left(\geqq-b^{2}\right)$. On désignera par $\Gamma$ le groupe des automorphismes de revêtement. Soit $\left(\widetilde{X}_{t}\right)_{t \in \mathbf{R}^{+}}$le brownien riemannien de $V$. Alors $X_{t}=\pi \circ \widetilde{X}_{t}$ est le brownien riemannien de $V$. On désignera par $P_{t}(x, y)$, resp. $\widetilde{P}_{t}(\widetilde{x}, \widetilde{y})$, la solution fondamentale de l'équation de la chaleur sur $V$, resp. $\widetilde{V}$.

Lemme 7. Pour $x, y \in V$, soient $\widetilde{x}, \tilde{y}$ des relèvements de $x$ et $y$ respectivement. On a:

$$
P_{t}(x, y)=\sum_{r \in \Gamma} \widetilde{P}_{t}(\widetilde{x}, \gamma \widetilde{y}) .
$$

Démonstration. Immédiate vue la relation entre les browniens.

Lemme 8. Pour $\widetilde{x}, \widetilde{y} \in \widetilde{V}$ et $t>0$, la série $\sum_{r \in \Gamma} e^{-d^{2}(\tilde{x}, r \tilde{y}) / 2 t}$ est sommable.

Posons $\Gamma_{k}=\{\gamma \in \Gamma / k \leqq d(\widetilde{x}, \gamma \widetilde{y})<k+1\}$. Un lemme démontré dans [3] et dû à Avez-Milnor assure que 
$\operatorname{Card}\left(\Gamma_{k}\right) \leqq C e^{(n-1) b(k+1)}$, où $C$ ne dépend pas se $\widetilde{x}, \widetilde{y}$.

On a évidemment

$\sum_{r \in \Gamma} \exp \left(-\frac{d^{2}(\widetilde{x}, \gamma \widetilde{y})}{2 t}\right)=\sum_{k=0}^{\infty} \sum_{r \in \Gamma_{k}} \exp \left(-\frac{d^{2}(\widetilde{x}, \gamma \widetilde{y})}{2 t}\right) \leqq \sum_{k=0}^{\infty} \exp \left(-\frac{k^{2}}{2 t}\right) \operatorname{Card}\left(\Gamma_{k}\right)$ d'où $\sum_{r \in \Gamma} \exp \left(-\frac{d^{2}(x, y)}{2 t}\right) \leqq c \sum_{k=0}^{\infty} \exp \left(-\frac{k^{2}}{2 t}\right) e^{(n-1) b(k+1)}$ et la série majorante est évidemment sommable, ce qui établit le lemme.

Dans la suite, $x_{0}, y_{0}$ seront deux points de $V$ fixés une fois pour toutes. Nous fixerons aussi un relèvement $\widetilde{x}_{0}$ de $x_{0}$. Tout relèvement de $y_{0}$, soit $\widetilde{y}_{0}$, satisfait $d\left(\widetilde{x}_{0}, \widetilde{y}_{0}\right) \geqq d\left(x_{0}, y_{0}\right)$, et il $y$ a au moins un $\widetilde{y}_{0} \in$ $\pi^{-1}\left(y_{0}\right)$ pour lequel l'égalité est atteinte. On suppose choisi une fois pour toutes un tel $\widetilde{y}_{0}$.

Un élément $\gamma$ de $\Gamma$ sera alors dit minimisant si $d\left(\widetilde{x}_{0}, \gamma \widetilde{y}_{0}\right)=d\left(x_{0}, y_{0}\right)$. L'élément identité de $\Gamma$ est évidemment minimisant.

Il existe une correspondance biunivoque entre les éléments de $\Gamma$ et les géodésiques de $V$ joignant $x_{0}$ à $y_{0}$ : à l'élément $\gamma$ est associée la géodésique de $V$ projection de l'unique géodésique de $\widetilde{V}$ qui joint $\widetilde{x}_{0}$ à $\gamma \widetilde{y}_{0}$. Dans la suite il nous arrivera d'identifier l'élément $\gamma$ avec la géodésique associée. Il est clair qu'aux éléments minimisants correspondent les géodésiques minimisantes. Ainsi, l'identité est le seul élément minimisant si et seulement si $y_{0}$ n'est pas dans le cut-locus de $x_{0}$.

On désignera par $M$ l'ensemble des éléments minimisants.

\section{Lemme 9.}

1) Si r n'est pas minimisant, on a

$$
\lim _{t \rightarrow 0} \frac{\widetilde{P}_{t}\left(\widetilde{x}_{0}, \gamma \widetilde{y}_{0}\right)}{P_{t}\left(x_{0}, y_{0}\right)}=0
$$

2) si $\Gamma$ est minimisant, $\frac{\widehat{P}_{t}\left(\widetilde{x}_{0}, \gamma \widetilde{y}_{0}\right)}{P_{t}\left(x_{0}, y_{0}\right)}$ reste minoré pour $t \rightarrow 0$ par un nombre strictement positif; si de plus $\widetilde{V}$ satisfait la condition (E) de Colin de Verdière, on $a$ :

$$
\lim _{t \rightarrow 0} \frac{\widetilde{P}_{t}\left(\widetilde{x}_{0}, \gamma \widetilde{y}_{0}\right)}{P_{t}\left(x_{0}, y_{0}\right)}=-\frac{\tilde{\theta}^{-1 / 2}\left(\widetilde{x}_{0}, \gamma \widetilde{y}_{0}\right)}{\sum_{\delta \in M} \widetilde{\theta}^{-1 / 2}\left(\widetilde{x}_{0}, \gamma \widetilde{y}_{0}\right)}
$$

où $\tilde{\theta}\left(\widetilde{x}_{0}.\right)$ est la fonction densité de volume riemannien de $V$ en coordonnées cartésiennes exponentielles d'origine $\widetilde{x}_{0}$. 
Démonstration.

1) $\quad \frac{\widetilde{P}_{t}\left(\widetilde{x}_{0}, \gamma \widetilde{y}_{0}\right)}{P_{t}\left(x_{0}, y_{0}\right)}=\left(\sum_{\delta \in \Gamma} \frac{\widetilde{P}_{t}\left(\widetilde{x}_{0}, \delta \widetilde{y}_{0}\right)}{\widetilde{P}_{t}\left(\widetilde{x}_{0}, \gamma \widetilde{y}_{0}\right)}\right)^{-1} \leqq\left(\sum_{\delta \in M} \frac{\widetilde{P}_{t}\left(\widetilde{x}_{0}, \delta \widetilde{y}_{0}\right)}{\widetilde{P}_{t}\left(\widetilde{x}_{0}, \gamma \widetilde{y}_{0}\right)}\right)^{-1}$.

Il suffit de montrer que chacun des termes de la dernière somme tend vers $+\infty$. Or, on a:

$$
\begin{aligned}
\frac{\tilde{P}_{t}\left(\widetilde{x}_{0}, \delta \widetilde{y}_{0}\right)}{\widetilde{P}_{t}\left(\widetilde{x}_{0}, \gamma \widetilde{y}_{0}\right)} \geqq \frac{\widetilde{P}_{t}^{\left(-b^{2}\right)}\left(\widetilde{x}_{0}, \delta \widetilde{y}_{0}\right)}{\widetilde{P}_{t}^{(0)}\left(\widetilde{x}_{0}, \gamma \widetilde{y}_{0}\right)} \\
\quad=\exp \left(\frac{d^{2}\left(\widetilde{x}_{0}, \gamma \widetilde{y}_{0}\right)-d^{2}\left(\widetilde{x}_{0}, \delta \widetilde{y}_{0}\right)}{2 t}\right) \widetilde{U}_{t}^{\left(-b^{2}\right)}\left(\widetilde{x}_{0}, \delta \widetilde{y}_{0}\right)
\end{aligned}
$$

avec $\widetilde{U}_{t}^{\left(-b^{2}\right)}(\widetilde{x}, \tilde{y})=(2 \pi t)^{n / 2} e^{d^{2}(\tilde{x}, \tilde{y}) / 2 t} \widetilde{P}_{t}^{\left(-b^{2}\right)}(\widetilde{x}, \widetilde{y})$.

Puisque $\delta$ est minimisant et $\gamma$ non, l'exponentielle tend vers $+\infty$, tandis que d'après le lemme $6, \widetilde{U}_{t}^{\left(-b^{2}\right)}\left(\widetilde{x}_{0}, \delta \widetilde{y}_{0}\right)$ a une limite strictement positive.

D'où le résultat.

2) Pour la première partie, il s'agit de montrer que $\sum_{\delta \in \Gamma} \frac{\widetilde{P}_{t}\left(\widetilde{x}_{0}, \delta \widetilde{y}_{0}\right)}{\widetilde{P}_{t}\left(\widetilde{x}_{0}, \gamma \widetilde{y}_{0}\right)}$ reste borné pour $0<t \leqq t_{0}$. Or

$$
\begin{aligned}
\sum_{\delta} \frac{\widetilde{P}_{t}\left(\widetilde{x}_{0}, \delta \widetilde{y}_{0}\right)}{\widetilde{P}_{t}\left(\widetilde{x}_{0}, \gamma \widetilde{y}_{0}\right)} \leqq \sum_{\delta} \frac{\widetilde{P}_{t}^{(0)}\left(\widetilde{x}_{0}, \delta \widetilde{y}_{0}\right)}{\widetilde{P}_{t}^{\left(-b^{2}\right)}\left(\widetilde{x}_{0}, \gamma \widetilde{y}_{0}\right)} \\
=\frac{1}{\widetilde{U}_{t}^{\left(-b^{2}\right)}\left(\widetilde{x}_{0}, \gamma \widetilde{y}_{0}\right)} \sum_{\delta \in \Gamma} \exp \left(\frac{d^{2}\left(\widetilde{x}_{0}, \gamma \widetilde{y}_{0}\right)-d^{2}\left(\widetilde{x}_{0}, \delta \widetilde{y}_{0}\right)}{2 t}\right)
\end{aligned}
$$

la convergence de la dernière série étant assurée par le lemme 8 .

D’après le lemme 6 , le facteur $\frac{1}{\widetilde{U}_{t}^{\left(-b_{0}\right)}\left(\widetilde{x}_{0}, \gamma \widetilde{y}_{0}\right)}$ reste borné. D’autre part, chaque terme de la série est fonction croissante de $t$, donc est majoré pour $t \leqq t_{0}$ par $\exp \left(\frac{d^{2}\left(\widetilde{x}_{0}, \gamma \widetilde{y}_{0}\right)-d^{2}\left(\widetilde{x}_{0}, \delta \widetilde{y}_{0}\right)}{2 t_{0}}\right)$. La série est donc majorée par la valeur qu'elle prend pour $t=t_{0}$, et au total $\sum_{\delta} \frac{\widetilde{P}_{t}\left(\widetilde{x}_{0}, \delta \widetilde{y}_{0}\right)}{\widetilde{P}_{t}\left(\widetilde{x}_{0}, \gamma \widetilde{y}_{0}\right)}$ est borné.

Supposons maintenant que $\widetilde{V}$ satisfait la condition (E). Ecrivons:

$$
\sum_{\delta \in \Gamma} \frac{\widetilde{P}_{t}\left(\widetilde{x}_{0}, \delta \widetilde{y}_{0}\right)}{\widetilde{P}_{t}\left(\widetilde{x}_{0}, \gamma \widetilde{y}_{0}\right)}=\sum_{\delta \in M}+\sum_{\delta \in M}
$$

et appelons $R(t)$ la deuxième somme.

$\mathrm{La}$ première somme vaut

$$
\sum_{\delta \in M} \frac{\widetilde{U}_{t}\left(\widetilde{x}_{0}, \delta \widetilde{y}_{0}\right)}{\widetilde{U}_{l}\left(\widetilde{x}_{0}, \gamma \widetilde{y}_{0}\right)} .
$$


La condition entraîne $\lim _{t \rightarrow 0} \widetilde{U}_{t}\left(\widetilde{x}_{0}, \delta \widetilde{y}_{0}\right)=\theta^{-1 / 2}\left(\widetilde{x}_{0}, \delta \widetilde{y}_{0}\right)$. La somme étant finie on a

$$
\lim _{t \rightarrow 0} \sum_{\delta \in M} \frac{\widetilde{P}_{t}\left(\widetilde{x}_{0}, \delta \widetilde{y}_{0}\right)}{\widetilde{P}_{t}\left(\widetilde{x}_{0}, \gamma \widetilde{y}_{0}\right)}=\frac{1}{\widetilde{\theta}^{-1 / 2}\left(\widetilde{x}_{0}, \delta \widetilde{y}_{0}\right)} \sum_{\delta \in M} \tilde{\theta}^{-1 / 2}\left(\widetilde{x}_{0}, \widetilde{y}_{0}\right) .
$$

Pour finir d'établir 2) il suffit donc de montrer que $R(t)$ tend vers 0 .

$$
\begin{aligned}
\text { Posons } u_{\delta}(t) & =\frac{\widetilde{P}_{t}\left(\widetilde{x}_{0}, \delta \widetilde{y}_{0}\right)}{\widetilde{P}_{t}\left(\widetilde{x}_{0}, \gamma \widetilde{y}_{0}\right)} \text {. On a } \\
u_{\delta}(t) & =\frac{\widetilde{U}_{t}\left(\widetilde{x}_{0}, \delta \widetilde{y}_{0}\right)}{\widetilde{U}_{t}\left(\widetilde{x}_{0}, \gamma \widetilde{y}_{0}\right)} \exp \left(\frac{d^{2}\left(\widetilde{x}_{0}, \gamma \widetilde{y}_{0}\right)-d^{2}\left(\widetilde{x}_{0}, \delta \widetilde{y}_{0}\right)}{2 t}\right) .
\end{aligned}
$$

Le quotient tend vers $\frac{\tilde{\theta}^{-1 / 2}}{\widetilde{\theta}^{-1 / 2}\left(\widetilde{x}_{0}, \delta \widetilde{y}_{0}\right)}\left(\widetilde{x}_{0}\right)$, et l'exponentielle vers 0 puisque $\gamma$ est minimisant et $\delta$ non.

D'après le théorème de Lebesgue il suffit donc de démontrer que $u_{\delta}(t)$ est majoré par le terme général d'une série convergente, uniformément en $\left.t \in] 0, t_{0}\right]$. Mais on a vu précédemment que pour $0<t \leqq t_{0}$ on avait

$$
u_{\delta} \leqq \text { Cte } \exp \left(\frac{d^{2}\left(\widetilde{x}_{0}, \gamma \widetilde{y}_{0}\right)-d^{2}\left(\widetilde{x}_{0}, \delta \widetilde{y}_{0}\right)}{2 t_{0}}\right)
$$

le second membre étant terme général d'une série convergente. D’où le résultat.

Nous pouvons alors établir un théorème analogue qu théorème 7 .

Théorème 8. Soit $V$ compacte $a ̀$ courbure $\leqq 0, x_{0}$ et $y_{0}$ deux points de $V, \widetilde{x}_{0}$ et $\widetilde{y}_{0}$ des relèvements de $x_{0}$ et $y_{0}$ tels que $d\left(\widetilde{x}_{0}, \widetilde{y}_{0}\right)$ $=d\left(x_{0}, y_{0}\right)$; soit $\left(X_{t}^{(\varepsilon)}, t \in[0,1]\right)$ le processus conditionné accéléré allant de $x_{0}$ à $y_{0}$ dans le temps 1 . Alors, quand $\varepsilon$ iend vers 0 , pour tout $t \in[0,1]$ la loi de la variable aléatoire $X_{t}^{\left({ }^{(\varepsilon)}\right.}$ a pour support limite l'ensemble des $\gamma(t)$, oì $\gamma$ décrit l'ensemble $M$ des géodésiques minimisantes allant de $x_{0}$ à $y_{0}$. Si de plus $\widetilde{V}$ satisfait la condition (E) de Colin de Verdière, la loi de $X_{t}^{\left({ }^{(\varepsilon)}\right.}$ converge étroitement vers la mesure atomique $\sum_{r \in M} a_{r} \delta_{r(t)}$ où $a_{r}$ est donné par

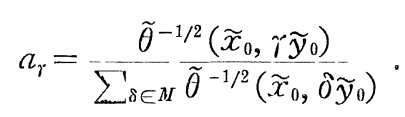




\section{Commentaires.}

1) Dans cer énoncé nous avons fait usage de l'identification précédemment signalée entre éléments de $\Gamma$ et géodésiques de $V$ allant de $x_{0}$ à $y_{0}$.

2) Quand nous écrivons que "la loi de $X_{t}^{(\varepsilon)}$ a pour support limite l'ensemble des $\gamma(t), \gamma \in M$ " nous entendons par là exactement ceci: appelons $\mu_{t}^{(\varepsilon)}$ cette loi; alors pour toute fonction $\Psi$ continue sur $V$ nulle sur l'ensemble considéré on a:

$$
\lim _{\varepsilon \rightarrow 0} \int_{V} \Psi(z) d \mu_{t}^{(\varepsilon)}(z)=0 ;
$$

d'autre part si $\Psi$ est une fonction continue sur $V$, à valeurs dans $[0,1]$, égale à 1 en l'un des points $\gamma(t)$, et nulle aux autres, alors $\int_{V} \Psi(z) d \mu_{t}^{\left({ }^{(\varepsilon)}\right.}(z)$ reste minoré pour $\varepsilon$ tendant vers 0 par une quantité strictement positive.

Démonstration. Soit $\Psi$ une fonction continue sur $V$. On pose $\widetilde{\Psi}^{\circ}$ $=\Psi \circ \pi$, de sorte que $\widetilde{\Psi}$ est une fonction continue bornée sur $\widetilde{V}$.

Supposons $\Psi$ nulle sur $\{\gamma(t)\}_{r \in M}$. Il nous faut alors montrer que

$$
\lim _{\varepsilon \rightarrow 0} \int_{V} \Pi_{i}^{(\varepsilon)}\left(x_{0}, z\right) \Psi(z) d z=0,
$$

soit $\lim _{\varepsilon \rightarrow 0} \int_{V} \frac{P_{\varepsilon t}\left(x_{0}, z\right) P_{\varepsilon(1-t)}\left(z, y_{0}\right)}{P_{\varepsilon}\left(x_{0}, y_{0}\right)} \Psi(z) d z=0$. On a :

$$
P_{\varepsilon t}\left(x_{0}, z\right) P_{\varepsilon(1-t)}\left(z, y_{0}\right)=\sum_{r \in \Gamma} \widetilde{P}_{\varepsilon t}\left(\widetilde{x}_{0}, \gamma \widetilde{z}\right) \sum_{\delta \in \Gamma} \widetilde{P}_{\varepsilon(1-t)}\left(\gamma \widetilde{z}, \delta \widetilde{y}_{0}\right)
$$

où $\widetilde{z}$ est un relèvement quelconque de $z$. Cela s'écrit encore (séries à termes $>0$ )

$$
P_{\varepsilon t}\left(x_{0}, z\right) P_{\varepsilon(1-t)}\left(z, y_{0}\right)=\sum_{r, \delta \in \Gamma} \widetilde{P}_{\varepsilon t}\left(\widetilde{x}_{0}, \gamma \widetilde{z}\right) \widetilde{P}_{\varepsilon(1-t)}\left(\gamma \widetilde{z}, \delta \widetilde{y}_{0}\right) .
$$

Dans la suite nous désignerons $B$ le domaine fondamental compact de $\Gamma$ contenant $\widetilde{x}_{0}$, tel que $\pi$ soit un difféomorphisme de l'intérieur de $B$ sur le complémentaire du cut-locus de $x_{0}$. On a alors:

$$
\begin{aligned}
& \int_{V} \frac{P_{\varepsilon t}\left(x_{0}, z\right) P_{\varepsilon(1-t)}\left(z, y_{0}\right)}{P_{\varepsilon}\left(x_{0}, y_{0}\right)} \Psi(z) d z \\
& \quad=\int_{B} \frac{1}{P_{\varepsilon}\left(x_{0}, y_{0}\right)}\left(\sum_{\gamma, \delta \in \Gamma} \widetilde{P}_{\varepsilon t}\left(\widetilde{x}_{0}, \gamma \widetilde{z}\right) \widetilde{P}_{\varepsilon(1-t)}\left(\gamma \widetilde{z}, \delta \widetilde{y}_{0}\right)\right) \widetilde{\Psi}(\widetilde{z}) d \widetilde{z}
\end{aligned}
$$


On peut supposer $\Psi \geqq 0$. On peut donc commuter $\sum$ et $\int$, et l'expression précédente devient:

$$
\sum_{\delta \in \Gamma} \frac{1}{P_{\varepsilon}\left(x_{0}, y_{0}\right)} \sum_{r \in \Gamma} \int_{B} \widetilde{P}_{\varepsilon t}\left(\widetilde{x}_{0}, \gamma \widetilde{z}\right) \widetilde{P}_{\varepsilon(1-t)}\left(\gamma \widetilde{z}, \delta \widetilde{y}_{0}\right) \widetilde{\Psi}(\gamma \widetilde{z}) d \widetilde{z}
$$

(puisqu'évidemment $\widetilde{\Psi}(\gamma \widetilde{z})=\widetilde{\Psi}(\widetilde{z})$ pour tout $\gamma \in \Gamma$.

Mais la série indexée par $\gamma$ est égale à $\int_{\widetilde{v}} \widetilde{P}_{\varepsilon l}\left(\widetilde{x}_{0}, \widetilde{z}\right) \widetilde{P}_{\varepsilon(1-t)}\left(\widetilde{z}, \delta \widetilde{y}_{0}\right)$ $\times \widetilde{\Psi}(\widetilde{z}) d \widetilde{z}$. Donc :

$$
\int_{V} \Pi_{t}^{(\varepsilon)}\left(x_{0}, z\right) \Psi(z) d z=\sum_{\delta \in \Gamma} \int_{\widetilde{v}} \frac{\widetilde{P}_{\varepsilon t}\left(\widetilde{x}_{0}, \widetilde{z}\right) \widetilde{P}_{\varepsilon(1-t)}\left(\widetilde{z}, \delta \widetilde{y}_{0}\right)}{P_{\varepsilon}\left(x_{0}, y_{0}\right)} \Psi(\widetilde{z}) d \widetilde{z}
$$

ce que l'on peut encore écrire:

$$
\sum_{\delta \in \Gamma} \frac{\widetilde{P}_{\varepsilon}\left(\widetilde{x}_{0}, \delta \widetilde{y}_{0}\right)}{P_{\varepsilon}\left(x_{0}, y_{0}\right)} \int_{\widetilde{r}} \frac{\widetilde{P}_{\varepsilon t}\left(\widetilde{x}_{0}, \widetilde{y}\right) \widetilde{P}_{\varepsilon(1-t)}\left(\widetilde{z}, \delta \widetilde{y}_{0}\right)}{\widetilde{P}_{\varepsilon}\left(\widetilde{x}_{0}, \delta \widetilde{y}_{0}\right)} \widetilde{\Psi}(\widetilde{z}) d \widetilde{z}
$$

Considérons individuellement les termes de la série en distinguant ceux qui correspondent à $\delta \in M$ et ceux qui correspondent à $\delta \notin M$.

Pour $\delta \in M$, soit $\tilde{\delta}$ le relèvement de la géodésique $\delta ; \tilde{\delta}$ joint $\widetilde{x}_{0}$ à $\delta \widetilde{y}_{0}$. Puisque $\Psi$ est nulle en $\delta(t), \widetilde{\Psi}$ est nulle en $\widetilde{\delta}(t)$. Alors d'après le théorème 7 , l'intégrale tend vers 0 . En ce qui concerne le facteur $\frac{\widetilde{P}_{\varepsilon}\left(\widetilde{x}_{0}, \delta \widetilde{y}_{0}\right)}{P_{\varepsilon}\left(x_{0}, y_{0}\right)}$, il reste $\leqq 1$. Donc les termes correspondant à $\delta \in M$ tendent vers 0 .

Pour $\delta \notin M$, le lemme 9 nous dit que le facteur $\frac{\widetilde{P}_{\varepsilon}\left(\widetilde{x}_{0}, \delta \widetilde{y}_{0}\right)}{P_{\varepsilon}\left(x_{0}, y_{0}\right)}$ tend vers 0. Quant à l'intégrale, elle est majorée par $\|\widetilde{\Psi}\|=\|\Psi\|$. Donc ces termes là aussi tendent vers 0 .

Pour prouver que la somme de la série tend vers 0 , il suffit alors de montrer qu'elle est majorée terme à terme indépendamment de $\delta$ par une série convergente. Or on a évidemment:

$$
\frac{\widetilde{P}_{\varepsilon}\left(\widetilde{x}_{0}, \delta \widetilde{y}_{0}\right)}{P_{\varepsilon}\left(x_{0}, y_{0}\right)} \leqq \frac{\widetilde{P}_{\varepsilon}\left(\widetilde{x}_{0}, \delta \widetilde{y}_{0}\right)}{\widetilde{P}_{\varepsilon}\left(\widetilde{x}_{0}, \widetilde{y}_{0}\right)}
$$

et comme on l'a vu dans la démonstration du lemme 9, le second membre de cette inégalité est majoré pour $\varepsilon \leqq \varepsilon_{0}$ par

$$
\text { Cte } \exp \left(\frac{d^{2}\left(x_{0}, y_{0}\right)-d^{2}\left(x_{0}, y_{0}\right)}{2 \varepsilon_{0}}\right)
$$

qui est le terme général d'une série convergente d'après le lemme 8 . 
On a donc établi que $\int_{V} \Pi_{t}{ }^{(\varepsilon)}\left(x_{0}, z\right) \Psi(z) d z$ tend vers 0 quand $\varepsilon$ tend vers 0 si $\Psi$ est nulle sur l'ensemble des $\gamma(t)$. Pour finir d'établir le théorème 8 , il faut maintenant considérer une fonction $\Psi$ continue à valeur dans $[0,1]$, égale à 1 en $\gamma(t)$ et à 0 aux autres points de l'ensemble, et montrer que pour une telle fonction

1) $\int_{V} \Pi_{t}{ }^{(\varepsilon)}\left(x_{0}, z\right) \Psi(z) d z$ reste minoré par une quantité strictement positive

2) si de plus $V$ satisfait la conditionnelle (E) de Colin de Verdière, $\int_{V} \Pi_{t}{ }^{(\varepsilon)}\left(x_{0}, z\right) \Psi(z) d z$ converge vers $\mathrm{a}_{r}$.

Or, on a évidemment

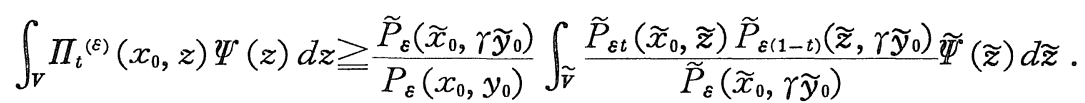

D'après le théorème 7 , l'intégrale tend vers $\widetilde{\Psi}(\widetilde{\gamma}(t))=\Psi(\gamma(t))=1$. Et d'après le lemme 9 le facteur $\frac{\widetilde{P}_{\varepsilon}\left(\widetilde{x}_{0}, \gamma \widetilde{y}_{0}\right)}{P_{\varepsilon}\left(x_{0}, y_{0}\right)}$ reste minoré au voisinage de $\varepsilon=0$ par un nombre strictement positif. Cela établit le 1).

Pour le 2) écrivons

$$
\begin{aligned}
\int_{V} \Pi_{t}{ }^{(\varepsilon)}\left(x_{0}, z\right)(z) & \left.d z=\frac{\widetilde{P}_{\varepsilon}\left(\widetilde{x}_{0}, \gamma \widetilde{y}_{0}\right)}{P_{\varepsilon}\left(x_{0}, y_{0}\right)} \int_{\widetilde{v}} \frac{\widetilde{P}_{\varepsilon t}\left(\widetilde{x}_{0}, \widetilde{z}\right) \widetilde{P}_{\varepsilon(1-t)}\left(\widetilde{z}, \gamma \widetilde{y}_{0}\right)}{\widetilde{P}_{\varepsilon}\left(\widetilde{x}_{0}, \gamma(\widetilde{z}) d \widetilde{z}\right.}\right) \\
& +\sum_{\delta \neq r} \frac{\widetilde{P}_{\varepsilon}\left(\widetilde{x}_{0}, \widetilde{y}_{0}\right)}{P_{\varepsilon}\left(x_{0}, y_{0}\right)} \int_{\widetilde{\Gamma}} \frac{\widetilde{P}_{\varepsilon t}\left(\widetilde{x}_{0}, \widetilde{z}\right) \widetilde{P}_{\varepsilon(1-t)}\left(\widetilde{z}, \delta \widetilde{y}_{0}\right) \widetilde{\Psi}(\widetilde{z}) d \widetilde{z}}{\widetilde{P}_{\varepsilon}\left(\widetilde{x}_{0}, \delta \widetilde{y}_{0}\right)}
\end{aligned}
$$

Comme dans la première partie de la démonstration, on voit que la série reste tend vers 0 . Quant au premier terme, l'intégrale tend vers 1, et le facteur qui est devant tend vers $a_{r}$ d'après le lemme 9. Cela établit le 2) et achève la démonstration du théorème 8.

\section{Références}

[1] Aubin, T., Espaces de Sobolev et inégalités isopérimétrique, Preprint (à paraître).

[2] Berger, M., Gauduchon, P. and Mazet, E., Le spectre d'une variété riemannienne, Springer Lecture Notes 194, 1971.

[3] Colin de Verdiere, Y., Spectre du laplacien et lonquers des géodésiques périodiques (I), Compositio Math. 27-1 (1973), 83-106.

[4] Debiard, A., Gaveau, B. and Mazet, E., Temps de sortie des boules normales et minoration locale de $\lambda_{1}$, Note C.R.A.S. Paris, 278 (1974) Ser A., 795-798.

[5] Doob, J. L., Boundary behaviour of functions with finite Dirichlet integrals, Ann. Inst. Fourier, 12 (1962), 573-626.

[6] Doob, J. L., Conditional brownian motion and the boundary limit of harmonic 
functions, Bull. Soc. Math. de France, 85 (1957), 431-458.

[7] Feynman, R. P., The Feynmann Lectures on Physics, tome III.

[8] Greene, R. E. and Wu, H., Curvature and complex analysis, Bull. Ainer. Math. Soc., 77 (1971), 1045-1049.

[9] Greene R. E. and Wu, H., A paraître aux inventiones mathematicae.

[10] Krein, M. G. and Rutman, M. A., Linear operators leaving invariant a cone in a Banach space, Amer. Math. Soc. Translations, série 1, 10 (1950), 199-325.

[11] Lichnerowicz, A., Géométrie des groupes de transformations, Dunod, Paris, 1958.

[12] McKean, H. P., An upper bound to the spectrum of $\Delta$ on a manifold of negative curvature, J. Differential Geometry, 4 (1970), 359-368.

[13] Malliavin, P., Asymptotics of the Green function on a riemannian manifold, Proc. Nat. Acad. Sci. U.S.A., 71-2 (1974), 381-383.

[14] Molchanov, A. S., Diffusion processes in Riemannian geometry, Upsekhi Mat. Nayk jan., (1975), (in Russian). English translation: Russian Math. Surveys, 30 (1975), $1-63$.

[15] Vauthier, J., Unicité de la solution de l'équation de la chaleur, Note CRAS Paris 1975-(28), p. 41. 
